\title{
Optimal Deterministic Auctions with Correlated Priors
}

\author{
Christos Papadimitriou* \\ UC Berkeley \\ christos@cs.berkeley.edu
}

\author{
George Pierrakos* \\ UC Berkeley \\ georgios@cs. berkeley.edu
}

\begin{abstract}
We revisit the problem of designing the profit-maximizing single-item auction, solved by Myerson in his seminal paper for the case in which bidder valuations are independently distributed. We focus on general joint distributions, either discrete or Lipschitz-continuous, seeking the optimal deterministic incentive compatible auction. We give a geometric characterization of the optimal auction, resulting in a duality theorem and an efficient algorithm for finding the optimal deterministic auction in the two-bidder case and an NP-completeness result for three or more bidders.
\end{abstract}

\section{Introduction}

Myerson's paper [21] on optimal auction design is remarkable in several ways. It is not the first important paper on auctions of course [31], but it pioneers the point of view of its title: auction design, that is, the exploration and evaluation of a large design space in a mindset that is very much one of computer science. It is elegant and methodologically original and powerful, completely resolving this important and difficult problem and providing insights, lemmata, and methodology that would be useful in related contexts for decades. Myerson considers the auction of an item to bidders with private valuations whose prior distributions are independent and known, and seeks incentive compatible mechanisms that maximize auctioneer revenue. His simple and elegant solution involves the virtual valuation, a quantity each bidder can compute from their private valuation and the valuation distribution through a mathematical maneuver called ironing. It follows that the auctioneer's and the bidders' actions in a given auction situation are easy to compute. Even though Myerson did not dwell on this aspect of his auction, it is clearly a sine qua non: no good auction design should involve solving intractable problems.

Myerson leaves open the case in which the valuations are correlated; in subsequent work, Crémer and McLean [8, 7] consider correlated valuations and solve the problem for the case where auctions are only required to be interim individually rational. In fact, in this framework the uncorrelated case is a singularity in the sense that in most cases (when the correlation has "full rank" in a certain precise sense) full surplus can be extracted in expectation through appropriate offers of lotteries to the bidders. Despite the elegance of their result, the fact that bidders may be charged merely for participating in the auction-lottery (including losers) has been criticized as rendering the auction impractical [20], especially for settings where agents may easily cancel their participation after the auction is conducted. It is therefore of tantamount importance to consider the question of designing the optimal ex post individually rational auction for correlated valuations. This is the question we consider in the current paper.

This past decade saw the advent and rise of Algorithmic Game Theory [24, 22], a research tradition which can be seen as a complexity-theoretic critique of Mathematical Economics, with Internet in the backdrop. This point of view has yielded a host of important results and new insights, for example related to the complexity of equilibria $[6,9]$, the trade-offs between complexity, approximation, and incentive-compatibility in social welfare-maximizing mechanism

${ }^{*}$ Research supported by NSF grant CCF-0964033 and by a Google University Research Award. 
design $[22,25]$, and (in an extended sense that includes on-line algorithms as a part of complexity theory) the price of anarchy $[19,30,29]$. However, there has been little progress in looking at Bayesian auctions à-la Myerson from this point of view. Ronen [27] came up with a mechanism for the correlated case that achieves half of the optimal revenue, while Ronen and Saberi [28] showed that no "ascending auction" can do better than $7 / 8$, and they conjectured that all relevant auctions are ascending (incidentally, we disprove this conjecture by showing that the optimal two-bidder auction may not be ascending). Missing from these two papers, however, is a concrete sense of the ways in which this is a difficult problem. We provide this here.

There were several follow-up papers $[4,17,10]$, examining the extent to which simple mechanisms can achieve good approximations of Myerson's optimal mechanism, and motivated by Myerson's astonishing result that the optimal mechanism for the regular i.i.d. setting is simply a second-price auction with reserve prices. For the most part, these are positive results yielding constant approximations by simple mechanisms in a variety of settings. Another line of work is prior-free mechanism design, where the goal is to design mechanisms that achieve profits comparable to that of some well-behaved benchmark [13]. This direction became especially interesting after Hartline and Roughgarden developed a framework in [16] that is grounded in Bayesian optimal mechanism design allowing one to design mechanisms that simultaneously approximate all Bayesian optimal mechanisms. The intermediate approach of having bidders' valuations coming from a distribution that is nonetheless unknown to the auctioneer has also been considered [10]. Finally the important open problem of designing optimal multi-parameter mechanisms has been addressed for some distributions of preferences, such as additive valuations with budget constraints [2] and unit-demand settings [5] and connections of this problem to the algorithmic problem of optimal pricing have been made [4, 14]; economists have also made some attempts to extend Myerson's results to multi-item auctions [1, 18].

In this paper we take a complexity-theoretic look at the general, correlated valuations case of Myerson's single-item auctions. We point out that the optimal auction design problem can be reduced essentially to a maximum weighted independent set problem in a particular graph whose vertices are all possible tuples of valuations (an uncountable set, of course, in the continuous case). If the distribution is discrete, this is an ordinary graph-theoretic problem; no such combinatorial characterization had been known, and this had been the main difficulty in developing an algorithmic and complexity-theoretic understanding of the problem. For discrete distributions, this leads directly to an efficient algorithm in the case of two bidders, where the graph is bipartite, while in the case of three or more bidders NP-completeness (in fact, inapproximability) prevails. For continuous distributions, we prove a duality characterization through a Monge-Kantorovich-like problem [12], and from this a fully polynomial approximation scheme for two bidders when the distribution is continuous enough and accessible through an oracle. As an aside, we also sketch in the last section a $2 / 3$ approximation for three bidders, improving the previously best known approximation of [27].

Our results rest on a geometric characterization of optimal deterministic auctions. An important element of our proof is the so-called marginal profit contribution function; it bears some similarities to Myerson's virtual valuation function [21], the most important of them being that they both admit a marginal revenue interpretation in the spirit of [3]. However, despite their similarities and their somewhat common derivation, marginal profit contribution functions are different from Myerson's virtual valuations in a number of ways: they only take positive values, they are not necessarily monotone and they do not admit a natural interpretation as valuations in some modified domain. One important ingredient of Myerson's approach to the design of optimal auctions is an analytical maneuver he calls ironing; Myerson uses ironing to transform a potentially non-monotone allocation rule into a monotone one, without hurting revenue. Our approach circumvents ironing by restricting the space of mechanisms explored; we achieve that by imposing an additional technical condition which limits the design space into a subset of all mechanisms, but one which still contains all the optimal mechanisms.

The work most related to ours is that of Dobzinski, Fu and Kleinberg [11], who also study the problem of designing the optimal auction for the correlated setting. They obtain a collection of very interesting results, which however are quite complementary to ours: Based, among others, on insights from [27], they arrive at efficient algorithms for computing the optimal randomized mechanism that is truthful in expectation, and a constant factor approximation of the optimal 
deterministic auction for any number of bidders.

\section{Preliminaries}

Imagine $n$ bidders seeking an indivisible good offered in auction. We assume that each bidder has a private valuation $v_{i}$ for the item and that bidders' valuations are drawn from some joint distribution over $[0,1]^{n}$ whose density function we denote by $\phi(\mathbf{v})$. We consider both discrete and continuous $\phi$. Discrete distributions are the source of the combinatorial insights underlying our approach, while continuous distributions provide continuity with the spirit and methodology of Myerson's paper, another important source of inspiration. In the continuous case, we follow Myerson in making the analytically convenient assumption that $\phi(x)>0$ for all $x \in[0,1]^{n}$. This is hardly a loss of generality, since a small minimum value on every point can be achieved by changing $\phi$ very little. In stating an algorithm for the two-dimensional continuous case (Section 5), we shall also assume that $\phi$ is Lipschitz-continuous ${ }^{1}$ and accessible through an oracle (in such a way that, for example, it can be approximately integrated over nice regions).

In the discrete case, let $\operatorname{Sup}(\phi)$ denote the finite support of the joint discrete distribution $\phi$. Then $\phi$ is presented as a finite set of $|\operatorname{Sup}(\phi)|(n+1)$-tuples of the form $\left(v_{1}^{i}, v_{2}^{i}, \ldots, v_{n}^{i}, \phi^{i}\right)$, one for each point $v^{i} \in \operatorname{Sup}(\phi)$, where $\phi^{i}$ is the probability mass concentrated at the point $\left(v^{i}\right)$ of the support.

We are interested in designing auctions that maximize the auctioneer's profit. Formally, an auction consists of an allocation rule denoted by $x_{i}(\mathbf{v})$, the probability of bidder $i$ getting allocated the item, and a payment rule denoted by $p_{i}(\mathbf{v})$ which is the price paid by bidder $i$. In this paper we focus our attention on deterministic mechanisms so that $x_{i}(\mathbf{v}) \in\{0,1\}$. Our goal is to maximize the auctioneer's expected profit $\sum_{i=1}^{n} \mathbb{E}_{\mathbf{v}}\left[p_{i}(\mathbf{v})\right]$.

We want our auction to be ex-post incentive compatible (IC) and individually rational (IR), with no positive transfers (NPT). These notions are defined as follows:

$$
\begin{aligned}
\text { IC: } \forall i, v_{i}, v_{i}^{\prime}, v_{-i}: v_{i} x_{i}\left(v_{i}, v_{-i}\right)-p_{i}\left(v_{i}, v_{-i}\right) \geq v_{i} x_{i}\left(v_{i}^{\prime}, v_{-i}\right)-p_{i}\left(v_{i}^{\prime}, v_{-i}\right) \\
\text { IR: } \forall i, v_{i}, v_{-i}: v_{i} x_{i}\left(v_{i}, v_{-i}\right)-p_{i}\left(v_{i}, v_{-i}\right) \geq 0 \\
\text { NPT: } \forall i, v_{i}, v_{-i}: p_{i}\left(v_{i}, v_{-i}\right) \geq 0
\end{aligned}
$$

We say that an allocation function $x_{i}\left(v_{i}, v_{-i}\right)$ is monotone if $v_{i} \geq v_{i}^{\prime}$ implies $x_{i}\left(v_{i}, v_{-i}\right) \geq$ $x_{i}\left(v_{i}^{\prime}, v_{-i}\right)$ for all $i, v_{-i}$. For the case of deterministic mechanisms that we are interested in, monotonicity implies that $x_{i}\left(v_{i}, v_{-i}\right)$ is a step-function. The threshold value of such a stepfunction allocation rule is set to be the minimum winning valuation for every bidder given the valuations of the other bidders: $t_{i}\left(v_{-i}\right)=\inf \left\{v_{i} \in[0,1] \mid x_{i}\left(v_{i}, v_{-i}\right)=1\right\}$. In complete analogy, in the discrete case $t_{i}\left(v_{-i}\right)=\min \left\{v_{i} \in \operatorname{Sup}_{i} \mid x_{i}\left(v_{i}, v_{-i}\right)=1\right\}$, where $\operatorname{Sup}_{i}$ is the support of bidder $i$. The following theorem provides a characterization of mechanisms for the setting we are interested in; a proof can be found in [23].

Theorem 2.1. A deterministic mechanism satisfies IC, IR and NPT if and only if the following conditions hold.

1. $x_{i}\left(v_{i}, v_{-i}\right)$ is monotone.

2. For all $i, v_{i}, v_{-i}$ we have

$$
p_{i}\left(v_{i}, v_{-i}\right)= \begin{cases}t_{i}\left(v_{-i}\right) & \text { if } x_{i}\left(v_{i}, v_{-i}\right)=1 \\ 0 & \text { if } x_{i}\left(v_{i}, v_{-i}\right)=0 .\end{cases}
$$

\section{The Geometry of Optimal Auctions}

Here we focus on the two-bidder case, and provide an alternative geometric interpretation of the mechanism design problem as a space partitioning problem. Our characterization holds for any number of bidders, with the appropriate generalizations and modifications; however we only

\footnotetext{
${ }^{1}$ A function $f$ is Lipschitz-continuous if there exists a constant $\lambda$ such that $|f(x)-f(y)| \leq \lambda|x-y|$ for all $x, y$.
} 
address the multi-dimensional case in Section 6, where we will use our geometric characterization to establish the inapproximability of the problem for 3 or more bidders.

We start by noting that the allocation function can be described in terms of a partition of the unit square (the space of all possible valuation pairs) into three regions: In region $A$ bidder 1 gets the item, in region $B$ bidder 2 gets the item and in region $C$ neither gets the item. The shape of those regions is restricted by monotonicity as follows (see Figure 1): Region $A$ is rightward closed, meaning that $(x, y) \in A$ and $x^{\prime}>x$ implies $\left(x^{\prime}, y\right) \in A$, while region $B$ is, analogously, upward closed. These regions are captured by their boundaries: Region $A$ 's boundary is a function $\alpha:[0,1] \mapsto[0,1]$ where for all $y \in[0,1] \alpha(y)=\inf \{x:(x, y) \in A\}$, and similarly for region $B$ and its boundary $\beta(x)$.

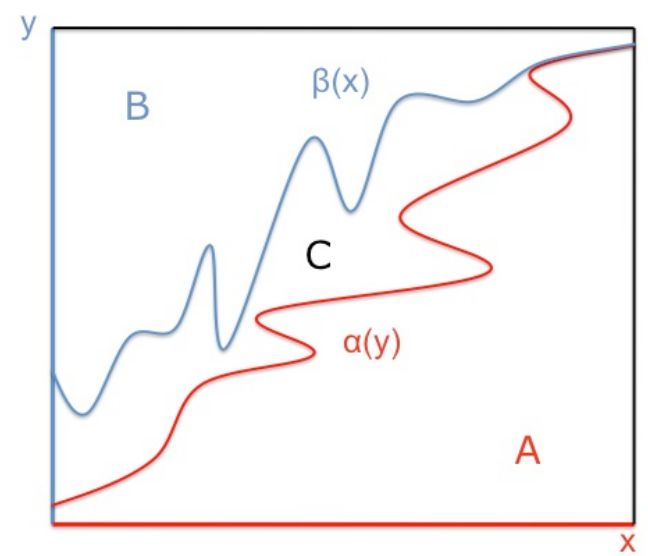

Figure 1: A pair of valid allocation rules.

Definition 3.1. A valid allocation pair $(\alpha, \beta)$ is a pair of functions from $[0,1]$ to itself satisfying the non-crossing property: for all points $(x, y) \in[0,1]^{2}$ we have $y \geq \beta(x) \Rightarrow x \leq \alpha(y)$.

Notice that it is not necessary for the functions to be monotone; the monotonicity property of the allocation is ensured by the fact that $\alpha$ and $\beta$ are proper functions and therefore regions $A$ and $B$ are rightward and upward closed respectively. The non-crossing property ensures that for any valuation pair $(x, y)$ at most one bidder is allocated the item.

In our proof we will make extensive use of the following notion of marginal profit.

Definition 3.2. Let $f$ (resp. $g$ ) be the marginal profit contribution of a valuation pair $(x, y)$ for bidder 1 (resp. 2), defined as:

$$
f(x, y)=-\frac{\partial}{\partial x}\left[\max _{x^{\prime} \geq x} x^{\prime} \cdot \int_{x^{\prime}}^{1} \phi(t, y) d t\right], g(x, y)=-\frac{\partial}{\partial y}\left[\max _{y^{\prime} \geq y} y^{\prime} \cdot \int_{y^{\prime}}^{1} \phi(x, t) d t\right]
$$

wherever the derivative is defined, and is extended to the full range by right continuity.

Intuitively, $f(x, y) d x d y$ is the added expected profit obtained from including the infinitesimal area $d x d y$ to $A$, that is, deciding to give the item to the first bidder if the valuations are $(x, y)$; in Remark 3.5 we further discuss the intuition behind these functions and their relation to Myerson's virtual valuation functions.

Definition 3.3. Call a valid allocation pair proper if it satisfies the following condition:

$$
\begin{aligned}
& \left.\alpha(y) \cdot \int_{\alpha(y)}^{1} \phi(t, y) d t=\max _{x^{\prime} \geq \alpha(y)} x^{\prime} \cdot \int_{x^{\prime}}^{1} \phi(t, y) d t, \quad \text { for all } y\right) \\
& \left.\beta(x) \cdot \int_{\beta(x)}^{1} \phi(x, t) d t=\max _{y^{\prime} \geq \beta(x)} y^{\prime} \cdot \int_{y^{\prime}}^{1} \phi(x, t) d t, \quad \text { for all } x\right\}
\end{aligned}
$$


Intuitively, in a proper valid pair $(\alpha, \beta)$, the curve $\alpha$ (resp. $\beta$ ) never goes through points that have zero marginal profit contribution $f(x, y)$ (resp. $g(x, y)$ ), as ensured by the first (resp. second) of the two equalities above.

Marginal profit contribution functions provide us with an alternative way to express the objective of expected profit.

Lemma 3.4. Let $(\alpha, \beta)$ be a proper valid allocation pair. Then the expected profit of any auction with payments defined as in Theorem 2.1 is:

$$
\int_{0}^{1} \int_{\alpha(y)}^{1} f(x, y) d x d y+\int_{0}^{1} \int_{\beta(x)}^{1} g(x, y) d y d x
$$

Proof. Let $p_{1}(x, y), p_{2}(x, y)$ be the payment functions induced by the allocation rule $(\alpha, \beta)$ according to Theorem 2.1. Then the expected profit of our auction is:

$$
\begin{aligned}
& \int_{0}^{1} \int_{0}^{1} p_{1}(x, y) \phi(x, y) d x d y+\int_{0}^{1} \int_{0}^{1} p_{2}(x, y) \phi(x, y) d x d y \\
= & \int_{0}^{1}\left[\alpha(y) \cdot \int_{\alpha(y)}^{1} \phi(t, y) d t\right] d y+\int_{0}^{1}\left[\beta(x) \cdot \int_{\beta(x)}^{1} \phi(x, t) d t\right] d x \\
= & \int_{0}^{1}\left[\max _{x^{\prime} \geq \alpha(y)} x^{\prime} \cdot \int_{x^{\prime}}^{1} \phi(t, y) d t\right] d y+\int_{0}^{1}\left[\max _{y^{\prime} \geq \beta(x)} y^{\prime} \cdot \int_{y^{\prime}}^{1} \phi(x, t) d t\right] d x \\
= & \int_{0}^{1} \int_{\alpha(y)}^{1} f(x, y) d x d y+\int_{0}^{1} \int_{\beta(x)}^{1} g(x, y) d y d x
\end{aligned}
$$

where in the first equality we used the characterization of truthful payments as every bidder's critical value, in the second equality we made use of condition (1) and in the last equality we made use of the definition of marginal profit contribution functions.

Remark 3.5. There is an intuitive connection between the marginal profit contribution and Myerson's (ironed) virtual valuations [21]. As pointed out in the introduction, even though these functions are not identical, the goal in both cases is the same: to capture some notion of marginal revenue. Indeed, the quantity $x \cdot \int_{x}^{1} \phi(t, y) d t$ corresponds to the expected profit of the auctioneer from an agent when she is offered a price of $x$, keeping the value of the other bidder fixed at $y$; equivalently, one can write the expected revenue as a function $R(q)$ of the probability of sale $q=1-\int_{x}^{1} \phi(t, y) d t$ (for a thorough discussion of this maneuver the reader is referred to [15]). Myerson's virtual valuations then correspond to the derivative of the function $R(q)$, and ironing corresponds to taking the convex hull $\hat{R}(q)$ of $R(q)$ and then differentiating. The "ironing" in our case corresponds to the action of taking the derivative of $R^{\prime}(q)=\max _{q^{\prime} \leq q} R\left(q^{\prime}\right)$ instead of $R(q)$, which is intuitively the "skyline" one would see from $q=0$. In Figure 2 we show an example of a revenue curve $R(q)$ and its corresponding $\hat{R}(q)$ and $R^{\prime}(q)$ curves.

It has been already noted by Myerson [21] that the allocation rule will remain invariant across ironed regions: in his case this is a consequence of his proof technique involving ironing. In our case we explicitly demand that the allocation is invariant by imposing Condition 1. Since the invariance of the allocation rule across ironed regions follows from Myerson's analysis as well, Condition 1 is indeed not a loss of generality in the sense that all optimal auctions satisfy it. We prove this formally next.

The next lemma establishes that without loss of generality we can restrict ourselves to proper allocation pairs. The economic intuition is the following: when two prices $p_{1}<p_{2}$ offered to bidder 1 result in the same expected profit (i.e. $p_{1} \int_{p_{1}}^{1} \phi(t, y) d t=p_{2} \int_{p_{2}}^{1} \phi(t, y) d t$ ), it is always to our advantage to charge bidder 1 a price of $p_{2}$, in order to be able to (potentially) allocate the item to bidder 2 for the valuation pairs $(x, y)$ with $x \in\left[p_{1}, p_{2}\right)$.

Let $\operatorname{Profit}(\alpha, \beta, \phi)$ denote the profit of an auction with allocation curves $(\alpha, \beta)$, when the joint distribution of valuations is $\phi$. 


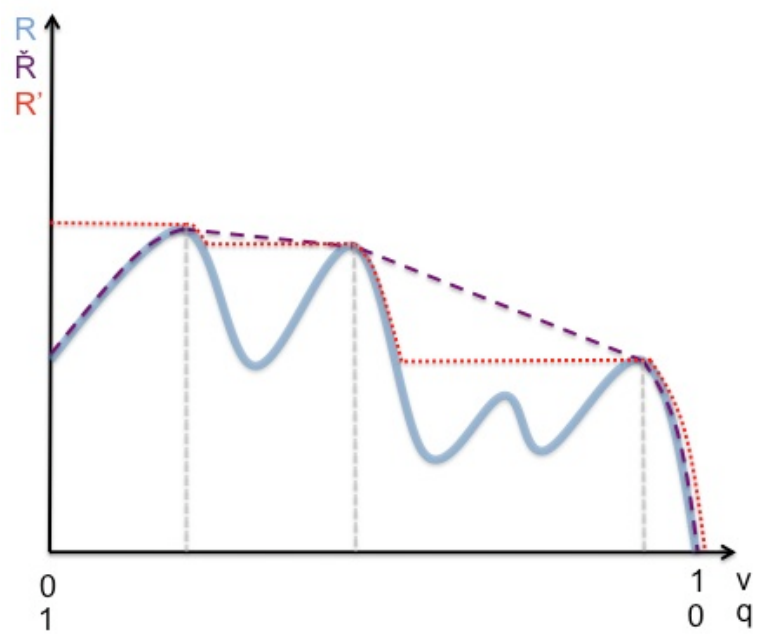

Figure 2: The revenue curves $R, \hat{R}$ and $R^{\prime}$.

Lemma 3.6. For any $\phi$ and for any valid allocation pair $(\alpha, \beta)$ there is a proper valid pair $\left(\alpha^{\prime}, \beta^{\prime}\right)$ such that Profit $\left(\alpha^{\prime}, \beta^{\prime}, \phi\right) \geq \operatorname{Profit}(\alpha, \beta, \phi)$

Proof. For the sake of contradiction suppose this is not true, i.e. Profit $\left(\alpha^{\prime}, \beta^{\prime}, \phi\right)<\operatorname{Profit}(\alpha, \beta, \phi)$ for any proper valid allocation pair $\left(\alpha^{\prime}, \beta^{\prime}\right)$.

We start by defining the following sets of points:

$$
\begin{aligned}
& \mathcal{Y}=\left\{y \in[0,1] \mid \alpha(y) \cdot \int_{\alpha(y)}^{1} \phi(t, y) d t \neq \max _{x^{\prime} \geq \alpha(y)} x^{\prime} \cdot \int_{x^{\prime}}^{1} \phi(t, y) d t\right\} \\
& \mathcal{X}=\left\{x \in[0,1] \mid \beta(x) \cdot \int_{\beta(x)}^{1} \phi(x, t) d t \neq \max _{y^{\prime} \geq \beta(x)} y^{\prime} \cdot \int_{y^{\prime}}^{1} \phi(x, t) d t\right\}
\end{aligned}
$$

as the set of all coordinates $y$ (resp. $x$ ) where condition (1) is violated by function $\alpha$ (resp. $\beta$ ). Consider now the auction defined by the following allocation curves:

$$
\begin{aligned}
& \alpha^{\prime}(y)= \begin{cases}\alpha(y) & , \text { if } y \notin \mathcal{Y} ; \\
\arg \max _{x^{\prime}}\left\{x^{\prime} \int_{x^{\prime}}^{1} \phi(t, y) d t \mid x^{\prime} \geq \alpha(y)\right\} & , \text { if } y \in \mathcal{Y} .\end{cases} \\
& \beta^{\prime}(x)= \begin{cases}\beta(x) & \text { if } x \notin \mathcal{X} ; \\
\arg \max _{y^{\prime}}\left\{y^{\prime} \int_{y^{\prime}}^{1} \phi(x, t) d t \mid y^{\prime} \geq \beta(x)\right\} & , \text { if } x \in \mathcal{X} .\end{cases}
\end{aligned}
$$

where - in the case of ties- $\arg \{\cdot\}$ returns the largest $y$ or $x$ respectively. By construction, the new pair $\left(\alpha^{\prime}, \beta^{\prime}\right)$ satisfies condition (1). In what follows we claim that the resulting allocation pair $\left(\alpha^{\prime}, \beta^{\prime}\right)$ is also valid and moreover it has greater revenue than $(\alpha, \beta)$, thus reaching a contradiction.

The monotonicity property of the allocation is satisfied since $\alpha^{\prime}$ and $\beta^{\prime}$ are proper functions of $y$ and $x$ respectively (see point following Definition 3.1). The non-crossing property follows from the non-crossing property of $\alpha$ and $\beta$ and the fact that $\alpha^{\prime}(y) \geq \alpha(y)$ for all $y \in[0,1]$ and $\beta^{\prime}(x) \geq \beta(x)$ for all $x \in[0,1]$. Finally, for the profit of the two auctions defined by the allocation 
curves $(\alpha, \beta)$ and $\left(\alpha^{\prime}, \beta^{\prime}\right)$ we have:

$$
\begin{aligned}
& \operatorname{Profit}\left(\alpha^{\prime}, \beta^{\prime}, \phi\right) \\
= & \int_{0}^{1}\left[\alpha^{\prime}(y) \cdot \int_{\alpha^{\prime}(y)}^{1} \phi(t, y) d t\right] d y+\int_{0}^{1}\left[\beta^{\prime}(x) \cdot \int_{\beta^{\prime}(x)}^{1} \phi(x, t) d t\right] d x \\
\geq & \int_{0}^{1}\left[\alpha(y) \cdot \int_{\alpha(y)}^{1} \phi(t, y) d t\right] d y+\int_{0}^{1}\left[\beta(x) \cdot \int_{\beta(x)}^{1} \phi(x, t) d t\right] d x \\
= & \operatorname{Profit}(\alpha, \beta, \phi),
\end{aligned}
$$

where the inequality follows from the definition of $\alpha^{\prime}$ and $\beta^{\prime}$. The lemma now follows.

Denote now by $\mathcal{A B}$ the set of all proper valid allocations $(\alpha, \beta)$. The problem of finding the optimal auction can now be restated as the following variational calculus-type problem:

\section{Definition 3.7. [Problem A]}

$$
\sup _{(\alpha, \beta) \in \mathcal{A B}}\left\{\int_{0}^{1} \int_{\alpha(y)}^{1} f(x, y) d x d y+\int_{0}^{1} \int_{\beta(x)}^{1} g(x, y) d y d x\right\}
$$

In this section we have established the following theorem:

Theorem 3.8. Finding the optimal auction for two bidders is equivalent to solving Problem A.

\section{Two Bidders: The Discrete Case}

In this section we present an algorithm for computing the optimal mechanism when there are two bidders with a discrete joint distribution $\phi$ with support $\operatorname{Sup}(\phi)=[N] \times[N]$.

The marginal profit contribution functions defined in the previous section can be appropriately modified for the discrete setting in hand:

Definition 4.1. The discrete analogues of the marginal profit contribution functions (Definition 3.2) for each bidder are defined as follows:

$$
\begin{aligned}
& f(i, j)=\max \left\{i \cdot \sum_{i^{\prime} \geq i} \phi\left(i^{\prime}, j\right)-\sum_{i^{\prime}>i} f\left(i^{\prime}, j\right), 0\right\} \text { for bidder } 1 \\
& g(i, j)=\max \left\{j \cdot \sum_{j^{\prime} \geq j} \phi\left(i, j^{\prime}\right)-\sum_{j^{\prime}>j} g\left(i, j^{\prime}\right), 0\right\} \text { for bidder } 2
\end{aligned}
$$

As in the continuous case, we will represent the auction through a pair of functions $(\alpha, \beta)$ :

Definition 4.2. A valid allocation pair $(\alpha, \beta)$ for the discrete setting is a pair of functions from $[N]$ to itself satisfying the non-crossing property: for all points $(i, j) \in[N] \times[N]$ we have $j \geq \beta(i) \Rightarrow i<\alpha(j)$. Such a pair partitions the set Sup $(\phi)$ into three sets $A, B$, and $C$, where $A=\{(i, j) \in \operatorname{Sup}(\phi): i \geq \alpha(j)\}, B=\{(i, j) \in \operatorname{Sup}(\phi): j \geq \beta(i)\}$, and $C=\operatorname{Sup}(\phi)-A-B$. We say that $(\alpha, \beta)$ induces the partition $(A, B)$, where $C$ is implicit.

Suppose that for a valid pair $(\alpha, \beta)$ representing an optimal auction and $j \in[n]$, we have that $f(\alpha(j), j)=0$. Then the auction represented by the same valid pair, except that $\alpha(j)$ is increased by one, is also an optimal auction (since it entails the same set of positive marginal profit contributions). We can therefore consider, without loss of generality, only valid pairs with $f(\alpha(j), j), g(i, \beta(i))>0$. We call such valid pairs proper. Finally, we define $\Pi(\phi)$ to be the set of all partitions $A, B$ of $\operatorname{Sup}(\phi)$ induced by proper valid pairs. 
Now, looking back at the formulation of the optimal revenue auction problem in Problem $A$ at the conclusion of the last section, it is immediate that obtaining the optimal revenue auction in the discrete case is tantamount to solving a discrete optimization problem:

$$
\max _{(A, B) \in \Pi(\phi)} \sum_{(i, j) \in A} f(i, j)+\sum_{(i, j) \in B} g(i, j)
$$

All that remains now is to provide a useful characterization of the set $\Pi(\phi)$. To this end, notice first that any such pair of sets $(A, B)$ has the following two additional properties, the first one inherited from incentive compatibility, and the second one following from the fact that the allocation curves form a proper valid pair:

Definition 4.3. We call a pair of disjoint subsets $(A, B)$ of $\operatorname{Sup}(\phi)$ monotone if the following holds:

- If $(i, j) \in A$ and $\left(i^{\prime}, j\right) \in \operatorname{Sup}(\phi)$ with $i^{\prime}>i$, then $\left(i^{\prime}, j\right) \in A$.

- If $(i, j) \in B$ and $\left(i, j^{\prime}\right) \in \operatorname{Sup}(\phi)$ with $j^{\prime}>j$, then $\left(i, j^{\prime}\right) \in B$.

We call such a pair proper if

- If $(i, j) \in A$ and $\left(i^{\prime}, j\right) \notin A$ for all $i^{\prime}<i$, then $f(i, j)>0$.

- If $(i, j) \in B$ and $\left(i, j^{\prime}\right) \notin B$ for all $j^{\prime}<j$, then $g(i, j)>0$. That is, in proper partitions, all lower boundary points of the regions $A$ and $B$ have positive marginal profit contributions (the intuition being that otherwise, either this is not an optimal auction in the case of a negative marginal profit contribution, or there is another optimal auction with this property in the case of a zero marginal profit contribution).

Let us define now a bipartite graph $G^{\phi}=(U, W, E)$ :

- $U=\left\{u_{i, j} \mid i, j \in[N]\right\}$ and $W=\left\{w_{i, j} \mid i, j \in[N]\right\}$;

- $\left(u_{i, j}, w_{i^{\prime}, j^{\prime}}\right) \in E$ if and only if $i \leq i^{\prime}$ and $j \geq j^{\prime}$. In other words, there is an edge between $u$ and $w$ if and only if, informally, $\left(i^{\prime}, j^{\prime}\right)$ "lies to the southeast" of $(i, j)$ (see Figure 3); (Notice that, in our informal sense, a point "lies to the southeast" of all points to its north and to its west, including the point itself.)

- The weight of any node $u_{i, j}$ is $f(i, j)$ and the weight of any node $w_{i, j}$ is $g(i, j)$.

Intuitively, the bipartite graph captures impossibilities in constructing the optimal auction: an edge $(u, w)$ signifies that it is not possible that both $u \in A$ and $w \in B$ (slightly abusing notation).

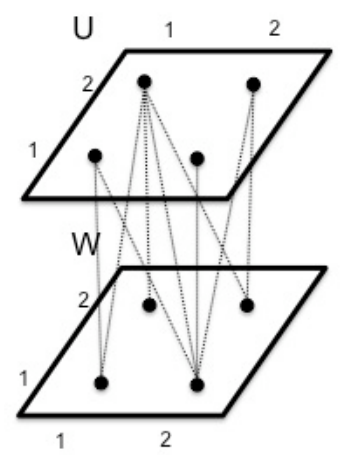

Figure 3: The bipartite graph for 2 bidders with $N=2$.

We can now prove the sought combinatorial characterization of $\Pi(\phi)$ :

Lemma 4.4. Let $(A, B)$ be a pair of disjoint subsets of $\operatorname{Sup}(\phi)$. Then $(A, B) \in \Pi(\phi)$ if and only if $(A, B)$ is monotone and proper, and $\left\{u_{i, j}:(i, j) \in A\right\} \cup\left\{w_{i, j}:(i, j) \in B\right\}$ is an independent set of $G^{\phi}$. 
Proof. (If.) Since $\left\{u_{i, j}:(i, j) \in A\right\} \cup\left\{w_{i, j}:(i, j) \in B\right\}$ is an independent set of $G^{\phi}$ it follows that the sets $A$ and $B$ are disjoint. Now, since $(A, B)$ is a pair of monotone, proper and disjoint subsets of $\operatorname{Sup}(\phi)$ the following pair of functions $(\alpha(j), \beta(i))$ is a proper valid pair, immediately implying that $(A, B) \in \Pi(\phi): \alpha(j)=\min \{i \mid(i, j) \in A\}$ and $\beta(i)=\min \{j \mid(i, j) \in B\}$.

(Only if.) If $(A, B) \in \Pi(\phi)$ then by the definition of $\Pi(\phi)$ the sets $(A, B)$ have to be monotone and proper and they also need to form a partition, i.e. be disjoint. To show that $\left\{u_{i, j}:(i, j) \in A\right\} \cup\left\{w_{i, j}:(i, j) \in B\right\}$ is an independent set of $G^{\phi}$ assume towards contradiction that there are nodes $u_{i, j}$ and $w_{i^{\prime}, j^{\prime}}$ such that $(i, j) \in A$ and $\left(i^{\prime}, j^{\prime}\right) \in B$, with an edge between $u_{i, j}$ and $w_{i^{\prime}, j^{\prime}}$; from the construction of $G^{\phi}$, it follows that $i \leq i^{\prime}$ and $j \geq j^{\prime}$. Since $A$ and $B$ are both monotone, it follows that $\left(i^{\prime}, j\right) \in A \cap B$, contradicting the disjointness of $A$ and $B$.

The next Lemma shows that the additional assumptions that $(A, B)$ is a proper and monotone pair of subsets are not necessary, if one restricts attention to the optimal solution (i.e. the solution of maximum weight).

Lemma 4.5. Let $(A, B)$ be a pair of subsets of $\operatorname{Sup}(\phi)$ such that the set $\left\{u_{i, j}:(i, j) \in A\right\} \cup\left\{w_{i, j}\right.$ : $(i, j) \in B\}$ is a maximum weight independent set of $G^{\phi}$, of minimum cardinality among all independent sets of the same weight. Then $(A, B)$ is monotone and proper, i.e. $(A, B) \in \Pi(\phi)$.

Proof. It suffices to show that the set $(A, B)$ is monotone and proper, and the lemma follows from Lemma 4.4. Indeed, the monotonicity of $(A, B)$ follows from the fact that the set $\left\{u_{i, j}\right.$ : $(i, j) \in A\} \cup\left\{w_{i, j}:(i, j) \in B\right\}$ is a maximum weight independent set, and all weights are nonnegative. Moreover, since the independent set has minimum cardinality among all independent sets of the same weight, it follows that it does not contain any node $u_{i, j}$ of zero weight, i.e. corresponding to some valuation $(i, j)$ such that $f(i, j)=0$, unless it also includes a node $u_{i^{\prime}, j}$ corresponding to some valuation $\left(i^{\prime}, j\right)$ with $f\left(i^{\prime}, j\right)>0$ for some $i^{\prime}<i$ (and analogously for $\left.w_{i, j}\right)$. Hence, by definition, $(A, B)$ is proper as well.

Theorem 4.6. Given a discrete joint valuation distribution $\phi$ for two bidders, the optimal ex-post IC and IR deterministic auction can be computed in time $O\left(|\operatorname{Sup}(\phi)|^{3}\right)$.

Proof. It follows from Lemma 4.5 that computing the optimal auction for two bidders with a joint valuation distribution $\phi$ reduces to computing a maximum weight independent set on the induced bipartite graph $G^{\phi}$. In particular, the optimal allocation rule corresponds to a partition $(A, B)$ such that $\left\{u_{i, j}:(i, j) \in A\right\} \cup\left\{w_{i, j}:(i, j) \in B\right\}$ is a maximum weight independent set of $G^{\phi}$, with minimum cardinality among all independent sets of the same weight. Finding the maximum weight independent set by running a min-cost-flow algorithm yields the desired running time.

\section{Two Bidders: The General Case}

In this section we return to the continuous two-bidder problem of Section 3. Our main result is an efficient algorithm that approximates the optimal solution of Problem $A$ within an arbitrarily small additive $\epsilon$. Our algorithm is quite natural: we first round the input, namely the bidders' distribution, in multiples of some constant $\epsilon$, and then run the maximum weight independent set algorithm on the resulting $1 / \epsilon \times 1 / \epsilon$ grid. The analysis however is more elaborate: we use as our main tool a duality theorem, generalizing the duality between the maximum-weight independent set problem in a bipartite graph and a minimum-cost flow in an associated network. In particular, we show that the maximization Problem $A$ defined in Section 3 is equivalent to a certain minimization problem, reminiscent in some aspects of the classic Monge-Kantorovich [12] mass-transfer problem, namely Problem $B$ defined below.

Problem $B$ is a continuous version of the min-cost flow dual of the maximum weighted independent set problem of the discrete case. "Continuous" here operates at many levels: The nodes of the bipartite graph (both sides thereof) are the points in the unit square; the edges of the bipartite graph are all possible directed edges going from one point in the unit square to another in the southeast direction. The capacities of the nodes are the values of the functions $f$ (left-hand side) and $g$ (right-hand side). 
Finally, one way of understanding the min-cost flow problem is the following: Suppose that the unit square is a garden of a particular geomorphology (hills, peaks and valleys, all above sea level) captured by the function $f$. We want to transform this landscape to the one captured by function $g$, and we want to do this by moving one grain of earth at a time. For each grain of earth in landscape $f$ we have two options: Either (1) we remove it, or (2) we move it in the southeast direction (or we keep it where it is, this counts as moving it a zero distance). We then complete our crafting of landscape $g$ by repeatedly (3) bringing in new grains. We want the plan in which the total amount of material moved (irrespective of distance moved, here is where this problem differs significantly from Monge-Kantorovich) is minimized.

Definition 5.1. [Problem B]

$$
\begin{array}{ll}
\inf _{\gamma} & \left\{\int_{0}^{1} \int_{0}^{1} \int_{0}^{y_{1}} \int_{x_{1}}^{1} \gamma\left(x_{1}, y_{1}, x_{2}, y_{2}\right) d x_{2} d y_{2} d x_{1} d y_{1}\right\} \\
\text { s.t. } & \int_{0}^{y_{1}} \int_{x_{1}}^{1} \gamma\left(x_{1}, y_{1}, x_{2}, y_{2}\right) d x_{2} d y_{2} \geq f\left(x_{1}, y_{1}\right), \forall\left(x_{1}, y_{1}\right) \in[0,1]^{2} \\
& \int_{y_{2}}^{1} \int_{0}^{x_{2}} \gamma\left(x_{1}, y_{1}, x_{2}, y_{2}\right) d x_{1} d y_{1} \geq g\left(x_{2}, y_{2}\right), \forall\left(x_{2}, y_{2}\right) \in[0,1]^{2} \\
& \gamma\left(x_{1}, y_{1}, x_{2}, y_{2}\right) \geq 0, \forall\left(x_{1}, y_{1}\right),\left(x_{2}, y_{2}\right) \in[0,1]^{2}
\end{array}
$$

Let us denote by $\Gamma$ the set of all functions $\gamma:[0,1]^{4} \mapsto \Re$ satisfying the above constraints. We next show that this problem coincides, at optimality, with the optimal auction:

Theorem 5.2. [Duality Theorem]

For any joint density function $\phi$ on $[0,1]^{2}$ :

$$
\begin{aligned}
& \sup _{(\alpha, \beta) \in \mathcal{A B}}\left\{\int_{0}^{1} \int_{\alpha(y)}^{1} f(x, y) d x d y+\int_{0}^{1} \int_{\beta(x)}^{1} g(x, y) d y d x\right\} \\
& =\inf _{\gamma \in \Gamma}\left\{\int_{0}^{1} \int_{0}^{1} \int_{0}^{y_{1}} \int_{x_{1}}^{1} \gamma\left(x_{1}, y_{1}, x_{2}, y_{2}\right) d x_{2} d y_{2} d x_{1} d y_{1}\right\}
\end{aligned}
$$

\subsection{Proof of the Duality Theorem}

General Plan. The proof of the theorem is by discretizing the unit square into domains of small size, proving a duality result for the discrete version, establishing upper bounds for the discretization error, and taking the limit for finer and finer discretization. In the course of the proof we will introduce a number of auxiliary problems.

Discretization. We start by discretizing the continuous functions $f$ and $g$ defined on $[0,1] \times$ $[0,1]$ by two discrete functions $f_{d}$ and $g_{d}$ defined on the $[n] \times[n]$ grid, where $n$ is an integer greater than one and $[\mathrm{n}]=\{0,1, \ldots, \mathrm{n}-1\}$; we denote $\frac{1}{n}$ by $\epsilon$. We subdivide the $[0,1] \times[0,1]$ square into $\epsilon \times \epsilon$ little squares; we are mapping a little square with southwest coordinate $(x, y)$ to the grid point $n \cdot(x, y)$. The discrete functions are now obtained by assigning to each point in the grid the aggregate mass of its corresponding square on the plane.

$$
f_{d}(i, j)=\int_{\epsilon i}^{\epsilon(i+1)} \int_{\epsilon j}^{\epsilon(j+1)} f(x, y) d y d x, \text { for } i, j=0, \ldots, n-1
$$

and

$$
g_{d}(i, j)=\int_{\epsilon i}^{\epsilon(i+1)} \int_{\epsilon j}^{\epsilon(j+1)} g(x, y) d y d x \text { for } i, j=0, \ldots, n-1 .
$$




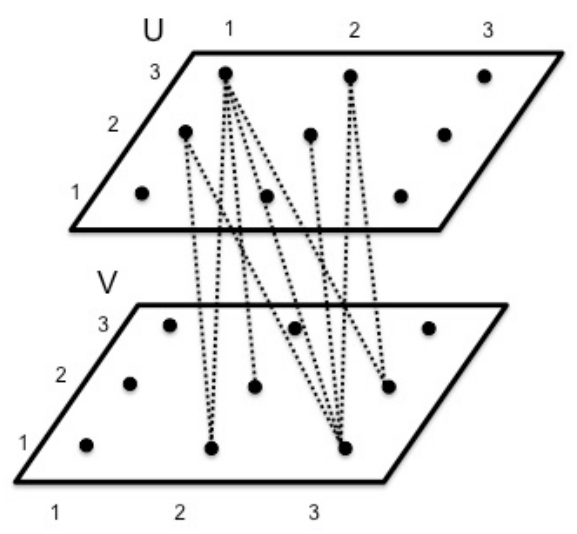

Figure 4: The graph consisting of two grids, one for each bidder, for $n=3$.

The Graph. We next create a weighted bipartite graph $G=(U, V, E)$ as follows: $U=V=$ $[n] \times[n]$. We use $u(i, j)$ and $v(i, j)$ to denote the vertices of $U$ and $V$ respectively, and sometimes use the shorthand $u$ and $v$ to refer to nodes of each grid respectively. Vertex $u(i, j)$ of $U$ has a weight equal to $w_{u}=f_{d}(i, j)$, and similarly vertex $v\left(i^{\prime}, j^{\prime}\right)$ of $V$ has a weight $w_{v}=g_{d}\left(i^{\prime}, j^{\prime}\right)$. A pair $\left(u(i, j), v\left(i^{\prime}, j^{\prime}\right)\right)$ is in $E$ if and only if $i<i^{\prime}$ and $j>j^{\prime}$, that is, if grid point $v$ is in the (strictly) southeast direction from grid point $u$ (see Figure 4 for an example with $n=3$ ).

Consider now the following problem, familiar from the previous section:

Definition 5.3. [Problem C: Maximum Weight Independent Set]

Given the weighted bipartite graph $G=(U, V, E)$ above,

$$
\begin{array}{cl}
\max _{\left\{x_{u} \in\{0,1\}, x_{v} \in\{0,1\}\right\}} & \sum_{u \in U, v \in V} x_{u} w_{u}+x_{v} w_{v} \\
\text { s.t. } & x_{u}+x_{v} \leq 1, \forall(u, v) \in E
\end{array}
$$

The dual of the above problem is the following:

Definition 5.4. [Problem D: Minimum Cost Transshipment]

Given the weighted bipartite graph $G=(U, V, E)$ above,

$$
\begin{array}{cl}
\min _{\left\{y_{u v} \in \Re\right\}} & \sum_{(u, v) \in E} y_{u v} \\
\text { s.t. } & \sum_{v:(u, v) \in E} y_{u v} \geq w_{u}, \forall u \in U \\
& \sum_{u:(u, v) \in E} y_{u v} \geq w_{v}, \forall v \in V \\
& y_{u v} \geq 0, \forall(u, v) \in E
\end{array}
$$

The Inequalities. The crux of the proof is a sequence of inequalities relating the various solutions and optimal solutions of these four problems. In what follows we use $S O L(\cdot)$ to denote the cost of a feasible solution of any of the problems defined above, and $O P T(\cdot)$ to denote the cost of the optimal solution of a problem (sometimes $S O L$ and $O P T$ also denote the actual solutions). The first such inequality establishes a form of weak duality between Problems $A$ and $B$, while the next two show that the discretization error is small.

Lemma 5.5. For any feasible solutions of $A$ and $B$ we have: $S O L(A) \leq S O L(B)$. 
Proof.

$$
\begin{aligned}
& \operatorname{SOL}(A) \\
= & \int_{0}^{1} \int_{\alpha\left(y_{1}\right)}^{1} f\left(x_{1}, y_{1}\right) d x_{1} d y_{1}+\int_{0}^{1} \int_{\beta\left(x_{2}\right)}^{1} g\left(x_{2}, y_{2}\right) d y_{2} d x_{2} \\
\leq & \int_{0}^{1} \int_{\alpha\left(y_{1}\right)}^{1} \int_{0}^{y_{1}} \int_{x_{1}}^{1} \gamma\left(x_{1}, y_{1}, x_{2}, y_{2}\right) d x_{2} d y_{2} d x_{1} d y_{1}+\int_{0}^{1} \int_{\beta\left(x_{2}\right)}^{1} \int_{y_{2}}^{1} \int_{0}^{x_{2}} \gamma\left(x_{1}, y_{1}, x_{2}, y_{2}\right) d x_{1} d y_{1} d y_{2} d x_{2}
\end{aligned}
$$

where we used the inequality constraints of Problem $B$ to upper bound the values of $f\left(x_{1}, y_{1}\right)$ and $g\left(x_{2}, y_{2}\right)$. We next notice that:

$\int_{0}^{1} \int_{\beta\left(x_{2}\right)}^{1} \int_{y_{2}}^{1} \int_{0}^{x_{2}} \gamma\left(x_{1}, y_{1}, x_{2}, y_{2}\right) d x_{1} d y_{1} d y_{2} d x_{2} \leq \int_{0}^{1} \int_{0}^{\alpha\left(y_{1}\right)} \int_{0}^{y_{1}} \int_{x_{1}}^{1} \gamma\left(x_{1}, y_{1}, x_{2}, y_{2}\right) d x_{2} d y_{2} d x_{1} d y_{1}$

This inequality follows from the non-negativity of $\gamma$ and the fact that the $\left(x_{1}, y_{1}, x_{2}, y_{2}\right)$ included in the integral of the LHS are the following set:

$$
\begin{aligned}
& \left\{\left(x_{1}, y_{1}, x_{2}, y_{2}\right) \in[0,1]^{4} \mid y_{2} \geq \beta\left(x_{2}\right), x_{1} \leq x_{2}, y_{1} \geq y_{2}\right\} \\
= & \left\{\left(x_{1}, y_{1}, x_{2}, y_{2}\right) \in[0,1]^{4} \mid y_{2} \geq \beta\left(x_{2}\right), x_{1} \leq x_{2}, y_{1} \geq y_{2}, x_{1} \leq \alpha\left(y_{1}\right)\right\} \\
\subseteq & \left\{\left(x_{1}, y_{1}, x_{2}, y_{2}\right) \in[0,1]^{4} \mid x_{2} \leq \alpha\left(y_{2}\right), x_{1} \leq x_{2}, y_{1} \geq y_{2}, x_{1} \leq \alpha\left(y_{1}\right)\right\} \\
\subseteq & \left\{\left(x_{1}, y_{1}, x_{2}, y_{2}\right) \in[0,1]^{4} \mid x_{1} \leq x_{2}, y_{1} \geq y_{2}, x_{1} \leq \alpha\left(y_{1}\right)\right\}
\end{aligned}
$$

which is exactly the set of $\left(x_{1}, y_{1}, x_{2}, y_{2}\right)$ included in the integral of the RHS. The first equality above follows from the fact that the inequality $x_{1} \leq \alpha\left(y_{1}\right)$ follows from the inequalities $\left\{y_{2} \geq\right.$ $\left.\beta\left(x_{2}\right), x_{1} \leq x_{2}, y_{1} \geq y_{2}\right\}$, because we have $y_{1} \geq \beta\left(x_{2}\right)$ so the non-crossing property implies that $x_{2} \leq \alpha\left(y_{1}\right)$ and therefore $x_{1} \leq \alpha\left(y_{1}\right)$. The first set inclusion follows from the fact that $y_{2} \geq \beta\left(x_{2}\right) \Rightarrow x_{2} \leq \alpha\left(y_{2}\right)$ from the non-crossing property, while the last inclusion is trivial.

We have therefore concluded that the cost of any feasible solution of $A$ is upper bounded by:

$$
\begin{aligned}
& \int_{0}^{1} \int_{\alpha\left(y_{1}\right)}^{1} \int_{0}^{y_{1}} \int_{x_{1}}^{1} \gamma\left(x_{1}, y_{1}, x_{2}, y_{2}\right) d x_{2} d y_{2} d x_{1} d y_{1}+\int_{0}^{1} \int_{0}^{\alpha\left(y_{1}\right)} \int_{0}^{y_{1}} \int_{x_{1}}^{1} \gamma\left(x_{1}, y_{1}, x_{2}, y_{2}\right) d x_{2} d y_{2} d x_{1} d y_{1} \\
= & \int_{0}^{1} \int_{0}^{1} \int_{0}^{y_{1}} \int_{x_{1}}^{1} \gamma\left(x_{1}, y_{1}, x_{2}, y_{2}\right) d x_{2} d y_{2} d x_{1} d y_{1} \\
= & S O L(B)
\end{aligned}
$$

Lemma 5.6. For the optimal solutions of $A$ and $C$ we have: $O P T(A) \geq O P T(C)-\epsilon$.

Proof. Consider the optimal solution of Problem $C$; we will use it to come up with a feasible solution for Problem $A$ such that $S O L(A) \geq O P T(C)-\epsilon$. We start with the following solution: we allocate the item to bidder 1 for all valuations $(x, y)$ such that $x_{u\left(\left\lfloor\frac{x}{\epsilon}\right\rfloor,\left\lfloor\frac{y}{\epsilon}\right\rfloor\right)}=1$; we allocate to bidder 2 for all valuations $(x, y)$ such that $x_{v\left(\left\lfloor\frac{x}{\epsilon}\right\rfloor,\left\lfloor\frac{y}{\epsilon}\right\rfloor\right)}=1$, and $x_{u\left(\left\lfloor\frac{x}{\epsilon}\right\rfloor,\left\lfloor\frac{y}{\epsilon}\right\rfloor\right)}=0$; and finally, we allocate to nobody for all valuations $(x, y)$ such that $x_{u\left(\left\lfloor\frac{x}{\epsilon}\right\rfloor,\left\lfloor\frac{y}{\epsilon}\right\rfloor\right)}=0$ and $x_{v\left(\left\lfloor\frac{x}{\epsilon}\right\rfloor,\left\lfloor\frac{y}{\epsilon}\right\rfloor\right)}=0$.

We next show that the resulting allocation regions have the shape of Figure 5, meaning that the borders of those regions consist a valid allocation pair. First notice that for any pair of valuations $(x, y)$-including those for which $x_{u\left(\left\lfloor\frac{x}{\epsilon}\right\rfloor,\left\lfloor\frac{y}{\epsilon}\right\rfloor\right)}=1$ and $x_{v\left(\left\lfloor\frac{x}{\epsilon}\right\rfloor,\left\lfloor\frac{y}{\epsilon}\right\rfloor\right)}=1$ - only one bidder gets allocated the item, so the non-crossing property is satisfied. To see why the regions are rightward and upward closed consider two nodes $u(i, j)$ and $u\left(i^{\prime}, j\right)$ on bidder 1's grid, where $i^{\prime}>i$. Notice that the set of nodes on bidder 2's grid that node $u(i, j)$ of bidder 1's grid is connected to, is a strict superset of the nodes that node $u\left(i^{\prime}, j\right)$ is connected to. Hence, if the maximum weight independent set includes node $u(i, j)$ on the grid of bidder 1 , it should also include $u\left(i^{\prime}, j\right)$ for all values $i^{\prime}>i$. 
This gives us two stairwise curves which -although being a valid allocation pair- may fail to satisfy Condition 1, and hence may not be a feasible solution for Problem $A$. To turn them into a proper valid pair, we can follow the same procedure as the one in the proof of Lemma 3.6 and come up with a feasible solution $\operatorname{SOL}(A)$ for Problem $A$.

Because of the aforementioned transformation the cost of this solution is greater or equal to the cost of the optimal solution $O P T(C)$ minus the contribution to the weight of the independent set by those nodes $v(i, j)$ for which the corresponding node $u(i, j)$ on the grid of bidder 1 is also included in the independent set. The reason for that is that for valuations $(x, y)$ such that $x_{v\left(\left\lfloor\frac{x}{\epsilon}\right\rfloor\left\lfloor\left\lfloor\frac{y}{\epsilon}\right\rfloor\right)\right.}=1$ and $x_{u\left(\left\lfloor\frac{x}{\epsilon}\right\rfloor,\left\lfloor\frac{y}{\epsilon}\right\rfloor\right)}=1$, our solution explicitly allocates the item only to bidder 1 , therefore losing the weight contribution of node $v\left(\left\lfloor\frac{x}{\epsilon}\right\rfloor,\left\lfloor\frac{y}{\epsilon}\right\rfloor\right)$. In what follows we argue that this results in the loss of an $\epsilon$-additive factor, so that the cost of the resulting solution is at least:

$$
\sum_{u \in U} x_{u} f_{d}(u)+\sum_{v \in V} x_{v} g_{d}(v)-\epsilon=O P T(C)-\epsilon
$$

To show this we first argue that the number of nodes $v(i, j)$ for which this happens is small, and in particular that there can only be at most $1 / \epsilon$ such nodes. To see this notice that in the constructed feasible solution to Problem $A$, these nodes lie on the boundary between regions where bidder 1 gets the item and bidder 2 gets the item; any such boundary has to be monotone, since it corresponds to the overlap of the two allocation curves $\alpha, \beta$, and it can therefore contain at most $1 / \epsilon$ nodes. Next notice that the value of $g$ at any point $(x, y)$ is at most 1 : indeed, $g(x, y)$ is defined as $y \phi(x, y)-\int_{y}^{1} \phi(x, t) d t$, wherever $\frac{\partial}{\partial y}\left[\max _{y^{\prime} \geq y} y^{\prime} \cdot \int_{y^{\prime}}^{1} \phi(x, t) d t\right]$ is defined, and extended to full range by right continuity. It follows immediately that $g(x, y) \leq 1$ and $w_{v(i, j)}=g_{d}(i, j) \leq \epsilon^{2}$; therefore the total weight loss is at most $\frac{1}{\epsilon} \cdot \epsilon^{2}$ and the lemma follows.

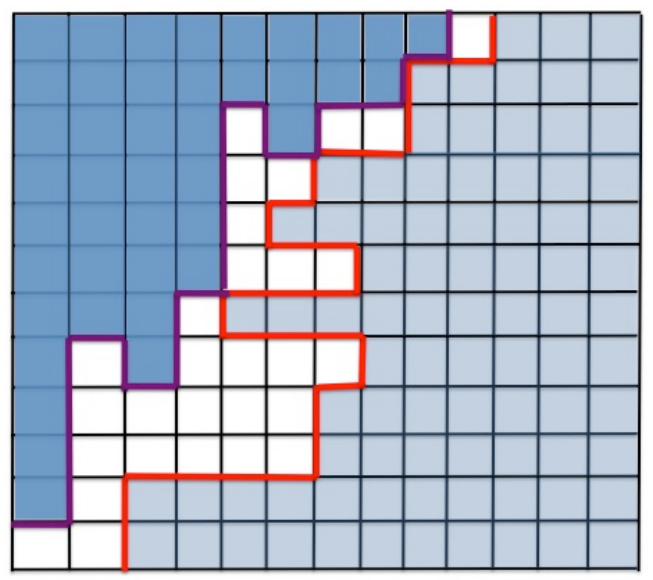

Figure 5: The solution of Problem $A$ (red and purple curves) resulting from the solution of Problem $C$ (blue and light-blue shaded regions) as described in Lemma 5.6.

Lemma 5.7. For the optimal solutions of $B$ and $D$ we have: $O P T(D) \geq O P T(B)$.

Proof. Given a feasible solution for Problem $D$, we will come up with a feasible solution of the same cost for Problem $B$. The optimal solution for Problem $B$ will have at most that cost and the lemma follows.

We start by defining $\gamma\left(x_{1}, y_{1}, x_{2}, y_{2}\right)$, for any pair of points $\left(x_{1}, y_{1}\right),\left(x_{2}, y_{2}\right)$ where the $\epsilon^{2}$-area square containing $\left(x_{2}, y_{2}\right)$ lies in the (strict) southeast orthant of the $\epsilon^{2}$-area square containing $\left(x_{1}, y_{1}\right)$, as follows:

$$
\gamma\left(x_{1}, y_{1}, x_{2}, y_{2}\right)=f\left(x_{1}, y_{1}\right) \cdot g\left(x_{2}, y_{2}\right) \cdot \frac{y_{u v}}{w_{u} w_{v}}, \quad \text { if }\left\lfloor\frac{x_{1}}{\epsilon}\right\rfloor<\left\lfloor\frac{x_{2}}{\epsilon}\right\rfloor \quad \text { and }\left\lfloor\frac{y_{1}}{\epsilon}\right\rfloor>\left\lfloor\frac{y_{2}}{\epsilon}\right\rfloor
$$


where $u \in U$ (resp. $v \in V)$ is the grid point $u\left(\left\lfloor\frac{x_{1}}{\epsilon}\right\rfloor,\left\lfloor\frac{y_{1}}{\epsilon}\right\rfloor\right)$ (resp. $\left.v\left(\left\lfloor\frac{x_{2}}{\epsilon}\right\rfloor,\left\lfloor\frac{y_{2}}{\epsilon}\right\rfloor\right)\right)$ that corresponds to the little $\epsilon^{2}$-area square containing point $\left(x_{1}, y_{1}\right)$ (resp. $\left.\left(x_{2}, y_{2}\right)\right)$. Finally, we let:

$$
\gamma\left(x_{1}, y_{1}, x_{2}, y_{2}\right)=0, \quad \text { if }\left\lfloor\frac{x_{1}}{\epsilon}\right\rfloor \geq\left\lfloor\frac{x_{2}}{\epsilon}\right\rfloor \quad \text { or }\left\lfloor\frac{y_{1}}{\epsilon}\right\rfloor \leq\left\lfloor\frac{y_{2}}{\epsilon}\right\rfloor
$$

We next verify that the function $\gamma$ defined above satisfies the constraints of Problem $B$. Since the non-negativity constraint is obviously satisfied, we only need to check that $\gamma$ satisfies the first and second constraints of Problem $B$. We only provide the proof for the first constraint and the proof for the second constraint follows along the exact same lines:

$$
\begin{aligned}
& \int_{0}^{y_{1}} \int_{x_{1}}^{1} \gamma\left(x_{1}, y_{1}, x_{2}, y_{2}\right) d x_{2} d y_{2}
\end{aligned}
$$

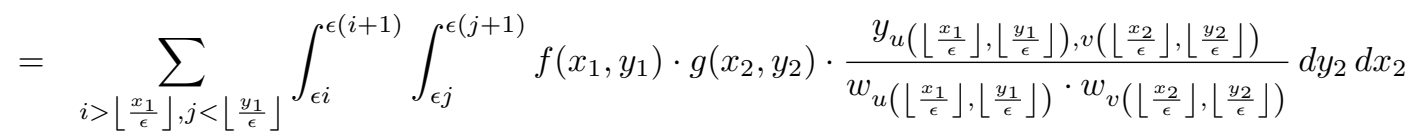

$$
\begin{aligned}
& =f\left(x_{1}, y_{1}\right) \cdot \sum_{i>\left\lfloor\frac{x_{1}}{\epsilon}\right\rfloor, j<\left\lfloor\frac{y_{1}}{\epsilon}\right\rfloor} \frac{y_{u\left(\left\lfloor\frac{x_{1}}{\epsilon}\right\rfloor\left\lfloor\left\lfloor\frac{y_{1}}{\epsilon}\right\rfloor\right), v(i, j)\right.}}{w_{u\left(\left\lfloor\frac{x_{1}}{\epsilon}\right\rfloor,\left\lfloor\frac{y_{1}}{\epsilon}\right\rfloor\right)} \cdot w_{v(i, j)}} \cdot \int_{\epsilon i}^{\epsilon(i+1)} \int_{\epsilon j}^{\epsilon(j+1)} g\left(x_{2}, y_{2}\right) d y_{2} d x_{2} \\
& =f\left(x_{1}, y_{1}\right) \cdot \sum_{i>\left\lfloor\frac{x_{1}}{\epsilon}\right\rfloor, j<\left\lfloor\frac{y_{1}}{\epsilon}\right\rfloor} \frac{y_{u\left(\left\lfloor\frac{x_{1}}{\epsilon}\right\rfloor,\left\lfloor\frac{y_{1}}{\epsilon}\right\rfloor\right), v(i, j)}}{w_{u\left(\left\lfloor\frac{x_{1}}{\epsilon}\right\rfloor\left\lfloor\frac{y_{1}}{\epsilon}\right\rfloor\right)} \cdot w_{v(i, j)}} \cdot w_{v(i, j)} \\
& =f\left(x_{1}, y_{1}\right) \cdot \sum_{i>\left\lfloor\frac{x_{1}}{\epsilon}\right\rfloor, j<\left\lfloor\frac{y_{1}}{\epsilon}\right\rfloor} \frac{y_{u\left(\left\lfloor\frac{x_{1}}{\epsilon}\right\rfloor,\left\lfloor\frac{y_{1}}{\epsilon}\right\rfloor\right), v(i, j)}}{w_{u\left(\left\lfloor\frac{x_{1}}{\epsilon}\right\rfloor,\left\lfloor\frac{y_{1}}{\epsilon}\right\rfloor\right)}} \\
& \geq f\left(x_{1}, y_{1}\right)
\end{aligned}
$$

where in the first equality we split the integration over discretized square regions of area $\epsilon^{2}$ (the same that are used in the discrete auxiliary Problems $C$ and $D$ ) and in the second equality we rearranged the order of summation and integration, noticing that the weights $w$ and flows $y$ remain constant across the discretized squares (independently of the actual value of $\left(x_{2}, y_{2}\right)$ ). In the third equality we used the definition of the weight $w$ and in the last inequality we used the fact that $y$ is a feasible solution for Problem $D$ and therefore $\sum_{v \in E} y_{u v} \geq w_{u}$.

We conclude our proof by showing that the cost of the feasible solution we produced is exactly $O P T(D)$ :

$$
\begin{aligned}
& \int_{0}^{1} \int_{0}^{1} \int_{0}^{y_{1}} \int_{x_{1}}^{1} \gamma\left(x_{1}, y_{1}, x_{2}, y_{2}\right) d x_{2} d y_{2} d x_{1} d y_{1} \\
& =\sum_{i, j} \int_{\epsilon i}^{\epsilon(i+1)} \int_{\epsilon j}^{\epsilon(j+1)} \int_{0}^{y_{1}} \int_{x_{1}}^{1} \gamma\left(x_{1}, y_{1}, x_{2}, y_{2}\right) d x_{2} d y_{2} d x_{1} d y_{1} \\
& =\sum_{i, j} \int_{\epsilon i}^{\epsilon(i+1)} \int_{\epsilon j}^{\epsilon(j+1)} f\left(x_{1}, y_{1}\right) \cdot \sum_{i^{\prime}>\left\lfloor\frac{x_{1}}{\epsilon}\right\rfloor, j^{\prime}<\left\lfloor\frac{y_{1}}{\epsilon}\right\rfloor} \frac{y_{u\left(\left\lfloor\frac{x_{1}}{\epsilon}\right\rfloor,\left\lfloor\frac{y_{1}}{\epsilon}\right\rfloor\right), v\left(i^{\prime}, j^{\prime}\right)}}{w_{u\left(\left\lfloor\frac{x_{1}}{\epsilon}\right\rfloor,\left\lfloor\frac{y_{1}}{\epsilon}\right\rfloor\right)}} d x_{1} d y_{1} \\
& =\sum_{i, j} \sum_{i^{\prime}>i, j^{\prime}<j} \frac{y_{u(i, j), v\left(i^{\prime}, j^{\prime}\right)}}{w_{u(i, j)}} \cdot \int_{\epsilon i}^{\epsilon(i+1)} \int_{\epsilon j}^{\epsilon(j+1)} f\left(x_{1}, y_{1}\right) d x_{1} d y_{1} \\
& =\sum_{i, j} \sum_{i^{\prime}>i, j^{\prime}<j} \frac{y_{u(i, j), v\left(i^{\prime}, j^{\prime}\right)}}{w_{u(i, j)}} \cdot w_{u(i, j)} \\
& =\sum_{i, j} \sum_{i^{\prime}>i, j^{\prime}<j} y_{u(i, j), v\left(i^{\prime}, j^{\prime}\right)} \\
& =O P T(D)
\end{aligned}
$$

where in the first equality we split the integration of $\left(x_{1}, y_{1}\right)$ over discretized square regions of area $\epsilon^{2}$ and in the second equality we plugged in the expression for $\int_{0}^{y_{1}} \int_{x_{1}}^{1} \gamma\left(x_{1}, y_{1}, x_{2}, y_{2}\right) d x_{2} d y_{2}$ 
that we had derived from our previous proof establishing that the first constraint of Problem $B$ was satisfied. In the third equality we once again rearranged the order of summation and integration, noticing that the weights $w$ and flows $y$ remain constant across the discretized squares (independently of the actual value of $\left.\left(x_{1}, y_{1}\right)\right)$, in the fourth equality we used the definition of the weight $w$ and in the last equality we replaced with the objective function of Problem $D$.

Proof of the Duality Theorem. First notice that, by strong duality and since the constraint matrix of Problem $C$ is totally unimodular, we have that $O P T(C)=O P T(D)$; combining this with Lemmas 5.6 and 5.7 we get $O P T(A) \geq O P T(B)-\epsilon$. From Lemma 5.5 we get $O P T(A) \leq O P T(B)$. By having $\epsilon \rightarrow 0$ we get the result.

\subsection{The Algorithm}

The proof of the Main Theorem suggests a fully polynomial-time approximation scheme (FPTAS) for the continuous case, that is, a mechanism that approximates the optimal profit within additive error $\epsilon$, and runs in time polynomial in $\frac{1}{\epsilon}$. In the algorithm and the correctness proof, we assume that the continuous joint distribution $\phi$ is Lipschitz continuous, and that it is presented through oracle access. It is easy to see that these assumptions are essentially necessary, in that no approximation (or meaningful solution of any other nature) is possible when the function can be arbitrarily discontinuous, or is inaccessible for large parts of the domain.

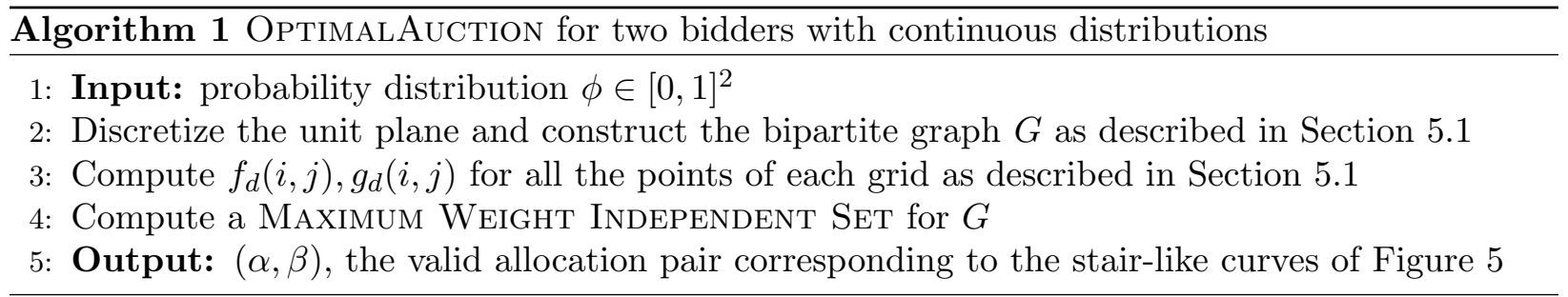

The following theorem establishes that our algorithm has the desired properties.

Theorem 5.8. Algorithm 1 returns a truthful mechanism that approximates the optimal profit within $\epsilon$ additive error; moreover the algorithm runs in time polynomial in both the support size and $1 / \epsilon$.

Proof. Algorithm 1 returns a valid allocation pair so it is truthful by construction. However the allocation pair $(\alpha, \beta)$ returned may well not satisfy condition (1) and may consequently not be a feasible solution to Problem $A$. This is problematic since it does not allow us to use Lemma 3.4 to compute the profit of the auction returned. To that end we need to establish that the violation of condition (1) is -in some sense- negligible; we do that next.

Suppose that curve $\alpha(y)$ violates condition (1) for some $y^{*} \in[0,1]$ and let

$$
x^{*}=\arg \max _{x \geq \alpha\left(y^{*}\right)} x \cdot \int_{x}^{1} \phi\left(t, y^{*}\right) d t,
$$

be the minimum $x$ for which we could create a new solution $\alpha^{\prime}$ by setting $\alpha^{\prime}\left(y^{*}\right)=x^{*}$ and have condition (1) restored, while not altering the profit of our auction. ${ }^{2}$ We will argue that

$$
\alpha\left(y^{*}\right) \cdot \int_{\alpha\left(y^{*}\right)}^{1} \phi\left(t, y^{*}\right) d t \geq x^{*} \cdot \int_{x^{*}}^{1} \phi\left(t, y^{*}\right) d t-\Theta(\epsilon)
$$

To do that we consider the node corresponding to the little square on the unit plane containing $\left(\alpha\left(y^{*}\right), y^{*}\right)$. Since this node belongs to the boundary of the allocation region of bidder 1 , we can assume wlog that it has non-zero weight; hence there must exist some point $\left(x_{1}, y_{1}\right)$ in

\footnotetext{
${ }^{2}$ The reader is referred to Lemma 3.6 for further discussion on this point.
} 
the corresponding square on the unit plane with $f\left(x_{1}, y_{1}\right)>0$. By the definition of $f$ this immediately implies that

$$
x_{1} \cdot \int_{x_{1}}^{1} \phi\left(t, y_{1}\right) d t \geq x \cdot \int_{x}^{1} \phi\left(t, y_{1}\right) d t, \quad \text { for all } x \geq x_{1}
$$

and therefore in particular that, if $x^{*} \geq x_{1}$ then

$$
x_{1} \cdot \int_{x_{1}}^{1} \phi\left(t, y_{1}\right) d t \geq x^{*} \cdot \int_{x^{*}}^{1} \phi\left(t, y_{1}\right) d t .
$$

Hence, if $x^{*} \geq x_{1}$, then, since the $l_{1}$-distance of points $\left(\alpha\left(y^{*}\right), y^{*}\right)$ and $\left(x_{1}, y_{1}\right)$ and of points $\left(x^{*}, y^{*}\right)$ and $\left(x^{*}, y_{1}\right)$ is at most $2 \epsilon$, we get that:

$\alpha\left(y^{*}\right) \cdot \int_{\alpha\left(y^{*}\right)}^{1} \phi\left(t, y^{*}\right) d t \geq x_{1} \cdot \int_{x_{1}}^{1} \phi\left(t, y_{1}\right) d t-2 \lambda \epsilon \geq x^{*} \cdot \int_{x^{*}}^{1} \phi\left(t, y_{1}\right) d t-2 \lambda \epsilon \geq x^{*} \cdot \int_{x^{*}}^{1} \phi\left(t, y^{*}\right) d t-4 \lambda \epsilon$

where in the first and third inequalities we used the fact that $x \cdot \int_{x}^{1} \phi(t, y) d t$ is Lipschitzcontinuous for some constant $\lambda$ and in the second inequality we used inequality (3).

If on the other hand $x^{*} \leq x_{1}$, then the points $\left(\alpha\left(y^{*}\right), y^{*}\right)$ and $\left(x^{*}, y^{*}\right)$ already have $l_{1}$-distance at most $2 \epsilon$ and it therefore follows immediately from Lipschitz continuity that:

$$
\alpha\left(y^{*}\right) \cdot \int_{\alpha\left(y^{*}\right)}^{1} \phi\left(t, y^{*}\right) d t \geq x^{*} \cdot \int_{x^{*}}^{1} \phi\left(t, y^{*}\right) d t-2 \lambda \epsilon .
$$

The exact same argument applies for $\beta(x)$ as well.

We are now ready to prove a lower bound on the profit of the auction returned by our algorithm; in what follows we use $\alpha^{\prime}(y)$ and $\beta^{\prime}(x)$ to denote the allocation curves that would result by the aforementioned transformation. Note that by construction it holds that

$$
\alpha^{\prime}(y) \cdot \int_{\alpha^{\prime}(y)}^{1} \phi(x, y) d x=\int_{\alpha^{\prime}(y)}^{1} f(x, y) d x \quad \text { and } \quad \beta^{\prime}(x) \cdot \int_{\beta^{\prime}(x)}^{1} \phi(x, y) d y=\int_{\beta^{\prime}(x)}^{1} g(x, y) d y
$$

so the profit of the algorithm is:

$$
\begin{aligned}
& \int_{0}^{1}\left[\alpha(y) \cdot \int_{\alpha(y)}^{1} \phi(x, y) d x\right] d y+\int_{0}^{1}\left[\beta(x) \cdot \int_{\beta(x)}^{1} \phi(x, y) d y\right] d x \\
\geq & \int_{0}^{1}\left[\alpha^{\prime}(y) \cdot \int_{\alpha^{\prime}(y)}^{1} \phi(x, y) d x-\Theta(\epsilon)\right] d y+\int_{0}^{1}\left[\beta^{\prime}(x) \cdot \int_{\beta^{\prime}(x)}^{1} \phi(x, y) d y-\Theta(\epsilon)\right] d x \\
= & \int_{0}^{1} \int_{\alpha^{\prime}(y)}^{1} f(x, y) d x d y+\int_{0}^{1} \int_{\beta^{\prime}(x)}^{1} g(x, y) d y d x-\Theta(\epsilon) \\
= & \operatorname{OPT}(C)-\Theta(\epsilon) \\
\geq & \operatorname{OPT}(A)-\Theta(\epsilon)
\end{aligned}
$$

where in the first inequality we used inequality (2), in the first equality we used (4) and in the last inequality we used our Main Theorem from the previous section.

In terms of running time, the discretized approximations of $f$ and $g$ are trivial (because of Lipschitz continuity, we can take $f_{d}(i, j)=f(i \epsilon, j \epsilon)$, and similarly for $g$ ). Solving the Maximum WEIGHT INDEPENDENT SET problem is done exactly as in the previous section.

\section{Three Bidders: NP-completeness}

In this section we show that, for three bidders, the problem of designing an approximately optimal (deterministic) auction becomes NP-hard, and in fact from 3SAT via approximationpreserving reductions. This implies that not only is the computational problem of designing the optimal deterministic auction intractable, but also that so is the problem of approximating this optimal auction within some finite relative error $\epsilon$. Finding a reasonably high value of the $\epsilon$ for which this statement holds is an interesting open problem. 


\subsection{A geometric reformulation}

We are interested in the complexity of the following problem:

Definition 6.1 (3OptimalAuctionDesign). Given a joint discrete probability distribution $\phi$ supported on $\mathcal{G}=[S] \times[S] \times[S]$ for some integer $S>0$, find the ex-post IC and IR deterministic auction, that is, the 3-dimensional allocation matrix $A$, where $A[x, y, z] \in\{0,1,2,3\}$, and $A[x, y, z]$ is the index of the bidder who gets the item when the valuation vector is $(x, y, z)$, or 0 if the auctioneer keeps the item, which maximizes revenue.

To calculate the revenue (last phrase of the previous definition), the following notion of marginal profit contribution, appropriately modified for the 3-bidder discrete case, will be useful to our proof.

Definition 6.2. The discrete analogues of the marginal profit contribution functions (Definition 3.2) for each bidder are the following:

$$
\begin{aligned}
& f(x, y, z)=\max \left\{x \cdot \sum_{x^{\prime} \geq x} \phi\left(x^{\prime}, y, z\right)-\sum_{x^{\prime}>x} f\left(x^{\prime}, y, z\right), 0\right\} \text { for bidder } 1 \\
& g(x, y, z)=\max \left\{y \cdot \sum_{y^{\prime} \geq y} \phi\left(x, y^{\prime}, z\right)-\sum_{y^{\prime}>y} g\left(x, y^{\prime}, z\right), 0\right\} \text { for bidder } 2 \\
& h(x, y, z)=\max \left\{z \cdot \sum_{z^{\prime} \geq z} \phi\left(x, y, z^{\prime}\right)-\sum_{z^{\prime}>z} h\left(x, y, z^{\prime}\right), 0\right\} \text { for bidder } 3
\end{aligned}
$$

The revenue corresponding to allocation $A$ is therefore

$$
\sum_{A(x, y, z)=1} f(x, y, z)+\sum_{A(x, y, z)=2} g(x, y, z)+\sum_{A(x, y, z)=3} h(x, y, z)
$$

The Segments. Given a distribution $\phi(x, y, z)$ supported on $\mathcal{G}$, any point $(x, y, z)$ of $\mathcal{G}$ with $f(x, y, z)>0$ is the apex of what we shall henceforth be calling an $x$-segment: a sequence of points starting at point $(x, y, z)$ and including all points $\left(x^{\prime}, y, z\right)$ with $x^{\prime} \geq x$. The weight of this segment is defined to be $\sum_{x^{\prime} \geq x} f\left(x^{\prime}, y, z\right)$, which, according to Definition 6.2, is equal to $x \cdot \sum_{x^{\prime} \geq x} \phi\left(x^{\prime}, y, z\right)$. We define segments across the other dimensions analogously. The following problem is equivalent to the auction design problem:

Definition 6.3 (3Segments). Given a joint discrete probability distribution $\phi$ supported on $\mathcal{G}$, which induces a set of segments on $\mathcal{G}$ as described above, find a subset of non-intersecting segments with maximum sum of weights.

Lemma 6.4. The problem $3 \operatorname{Segments}(\phi)$ is equivalent to 3 -OptimalAuctionDesign $(\phi)$ (via approximation-preserving reductions).

Proof. (Sketch:) The correspondence between solutions of the two problems is straightforward, illustrated in Figure 6.

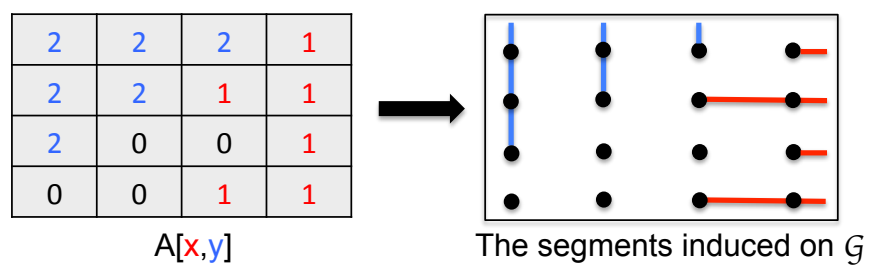

Figure 6: The reduction of Lemma 6.4, shown here for 2 bidders for ease of depiction. 


\subsection{The reduction}

We must therefore show that 3SEGMENTS is APX-hard; we prove that by reducing from TYPED3SAT, a special case of MAX3SAT:

Definition 6.5. Let TYPED3SAT be the MAX3SAT problem with the input formula restricted to be of the following form. We have three types of variables $\left\{x_{i}\right\}_{i=1 \ldots n_{x}},\left\{y_{i}\right\}_{i=1 \ldots n_{y}}$ and $\left\{z_{i}\right\}_{i=1 \ldots n_{z}}$, i.e. a total of $n=n_{x}+n_{y}+n_{z}$ variables, and $m$ clauses of the following form: every clause has at most one literal from every type (e.g. $\left(\bar{x}_{2} \vee \bar{y}_{3} \vee z_{1}\right)$ or $\left.\left(\bar{x}_{5} \vee z_{7}\right)\right)$. Moreover, every variable appears at most 7 times in the formula.

Lemma 6.6. TyPed 3 SAT is APX-complete.

Proof. We reduce from the 3SAT-5 problem, which is 3SAT with the additional constraint that every variable appears at most 5 times (this was shown in [26] to be APX-complete). In order to turn an instance $\Phi$ of 3 SAT- 5 to an equivalent instance $\Psi$ of TYPED3SAT, for every variable $x$ we create three copies $x_{1}, x_{2}, x_{3}$, and add the consistency clauses $\left(\bar{x}_{1} \vee x_{2}\right),\left(\bar{x}_{2} \vee x_{3}\right),\left(\bar{x}_{3} \vee x_{1}\right)$ to guarantee that all copies of the variable $x$ will have the same value. We then consider an arbitrary ordering of the variables within each clause, and replace any occurrence of variable $x$ at position $i \in\{1,2,3\}$ within a clause with $x_{i}$. Since a variable $x$ can appear at most 5 times in the original formula, each one of the copies $x_{1}, x_{2}, x_{3}$ can appear at most 7 times: 5 times in the original formula, if all occurrences of $x$ happen to be at the same position, and 2 times in the consistency clauses.

NP-hardness is immediate: Any satisfying truth assignment of the original formula yields immediately a satisfying truth assignment of the resulting formula, and vice-versa. To show that the reduction is approximation-preserving, suppose that we can satisfy a fraction of $(1-\epsilon)$ of $\Psi$. We claim that we can satisfy a fraction of $(1-50 \cdot \epsilon)$ of the original $\Phi$. To see this, notice that $\Psi$ has $m+3 n \leq 10 m$ clauses, and if an $\epsilon$ fraction of them is unsatisfied, or at most $10 \epsilon m$, this can affect at most $50 \mathrm{\epsilon m}$ of the $m$ clauses of $\Phi$. This is because each clause of $\Psi$ either is itself a clause of $\Phi$, or it is a consistency clause for some variable, which therefore affects the at most five clauses in $\Phi$ in which the variable appears. Therefore, the remaining clauses of $\Phi$ are satisfied.

\subsubsection{Overview of the construction}

The instance of 3SEGMENTS we create has three types of segments:

- Literal segments capture the truth assignment to variables; they ensure that every variable is assigned exactly one of the two possible truth values and that this assignment is consistent across all appearances of literals of this particular variable.

- Clause segments capture the truth assignment to literals of a particular clause; we create one such clause segment for every literal that appears in a clause. These segments intersect with each other and with some of the literal segments corresponding to those literals. As a result, if the clause is satisfied with the truth assignment implied by the literal segments, we will be able to pick at least one clause segment per clause (because the literal segment that intersects it will not be picked). Moreover, we cannot pick two or more clause segments per clause, since they will all intersect with each other, so we will end up picking exactly one such clause segment per satisfied clause.

- The scaffolding segments complete the construction in a way outlined later.

Suppose that the instance of Trped3SAT contains the clause $C=\left(x_{1} \vee y_{1} \vee \bar{z}_{1}\right)$. The corresponding 3SEGMENTS gadget is presented in Figure 7, where literal segments are depicted in red and clause segments are depicted in blue. There are two intersecting literal segments $\alpha$ and $\bar{\alpha}$ for each variable $\alpha$ (the intersections of segments are denoted for clarity as small circles); as only one of these can be selected in the solution (and it will be clear that one will be selected in the optimal solution), the solution will imply a truth assignment. 
Conversely, for any truth assignment we pick a set of literal segments for our 3SEGMENTS solution as follows: if the truth assignment sets $x_{1}=T R U E$ we are going to pick the literal segment labeled $x_{1}$ in Figure 7, otherwise, if $x_{1}=F A L S E$, we are going to pick the literal segment labeled $\bar{x}_{1}$. Notice each type of variables is assigned its own axis in 3-dimensional space. Notice also how the fact that these two segments intersect ensures the consistency of the assignment. The idea now is that any truth assignment that satisfies the clause, i.e. it sets at least one of the literals $x_{1}, y_{1}$ or $\bar{z}_{1}$ to true, results in a solution of 3SEGMENTs that includes at least one of the literal segments labeled $x_{1}, y_{1}, \bar{z}_{1}$. Therefore, the solution does not include at least one of the complementary segments labeled $\bar{x}_{1}, \bar{y}_{1}, z_{1}$; this allows us to include one of the blue clause segments to our 3SEGMENTS solution. In fact, we can include at most one blue segment corresponding to clause $C$, because the three blue segments associated with it intersect.

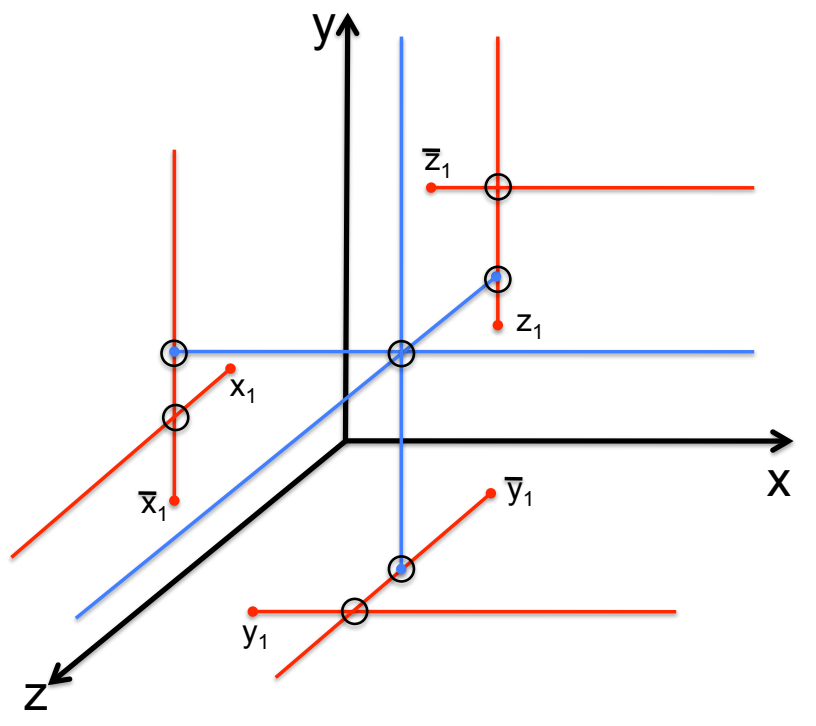

Figure 7: The gadget of 3SEGments resulting from the single clause $\left(x_{1} \vee y_{1} \vee \bar{z}_{1}\right)$ of Typed3SAT. This picture is not accurate -especially with respect to the location of the segments' apices- and is solely meant to convey the basic idea of the reduction. We give a precise example in the next section.

There are many details missing, of course. For example, recall that an instance of 3SEGMENTS is a probability distribution $\phi$, which induces the aforementioned segments as explained in the paragraph preceding Definition 6.3. Intuitively, this distribution should assign non-zero probability mass only at the points at which we want our segments to begin.

This leads to a technical complication, which necessitates the third kind of segments, the scaffolding segments: consider for simplicity the two-dimensional example of Figure 8, where the distribution is supported on the set $\{1,2,3\}^{2}$ and our goal is to include the blue segment depicted. We can achieve this by assigning some non-zero probability mass at point $(1,1)$, say $\phi(1,1)=1$, which will make $g(1,1)=1>0$ and will indeed induce the blue segment; however, since $f(1,1)=1>0$ as well, the instance will also induce the undesired red segment. To resolve this issue we introduce a scaffolding segment: we split the probability mass between points $(1,1)$ and $(3,1)$, for example $\phi(1,1)=\phi(3,1)=0.5$, so that $f(1,1)=0$ and the undesired red segment disappears. The new green segments starting at $(3,1)$ because of $f(3,1)=1.5, g(3,1)=0.5$, are what we refer to as scaffolding segments. 
(a)

\begin{tabular}{|l|l|l|l|}
\hline 3 & 0 & 0 & 0 \\
\hline 2 & 0 & 0 & 0 \\
\hline 1 & 1 & 0 & 0 \\
\hline & 1 & 2 & 3 \\
\hline & $\bullet$ & $\bullet$ \\
\hline
\end{tabular}

$\varphi$

The segments induced on $\mathcal{G}$

(b)

\begin{tabular}{|c|c|c|c|}
\hline 3 & 0 & 0 & 0 \\
\hline 2 & 0 & 0 & 0 \\
\hline 1 & 0.5 & 0 & 0.5 \\
\hline & 1 & 2 & 3 \\
\hline \multicolumn{4}{|c|}{$\varphi$}
\end{tabular}

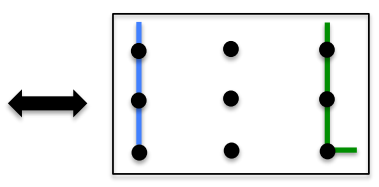

The segments induced on $G$

Figure 8: The usage of scaffolding segments.

\subsubsection{The detailed construction}

In this section we give the full details of the reduction, showing how to construct a probability distribution $\phi$ that serves as the input of 3SEGMENTs, given an instance of TrPED3SAT. We conclude with a worked out example.

Let $\hat{n}$ denote $\max \left\{n_{x}, n_{y}, n_{z}\right\}$. The support ${ }^{3}$ of $\phi$ is: $\{s(i) \mid i=1, \ldots, \hat{n}+2 m+4\}^{3}$, for an appropriate choice of the values $s(i)$ which we will fix later; for now all we assume is that $s$ is an increasing function of $i$. The size of the support is at most $(\hat{n}+2 m+4)^{3}$, so this is clearly a polynomial time construction.

In what follows we shall abuse notation and write $\phi(x, y, z)$ instead of $\phi(s(x), s(y), s(z))$ when there is no ambiguity. Also, in terms of phrasing, we shall sometimes refer to the submatrices $\phi(i, \cdot, \cdot), \phi(\cdot, j, \cdot), \phi(\cdot, \cdot, k)$ as the "planes" $x=i, y=j$ and $z=k$ respectively; finally, remember that a segment that runs parallel to the $x$ (resp. $y$ or $z$ ) axis is an $x$-segment (resp. $y$-segment or $z$-segment).

The construction proceeds as follows: Consider an arbitrary ordering of the clauses 1 through $m$ and suppose the $l$-th clause is of the form $\left(x_{i} \vee y_{j} \vee z_{k}\right)$ where $x_{i}, y_{j}$ and $z_{k}$ can be either positive or negative literals, and $i$ (resp. $j, k)=1, \ldots, n_{x}$ (resp. $n_{y}, n_{z}$ ) (the same construction also works for clauses with less than 3 variables). For this clause we are going to introduce the set of literal segments and clause segments described below.

Literal segments. To formally define the literal segments we first need to set the probability mass of the point that is the apex of each segment, and then use scaffolding segments to ensure that there is exactly one segment starting at each such point, towards the appropriate direction. We defer the discussion of scaffolding segments to a subsequent section, and here we only show which points are picked as apices.

In particular, the probability mass that is assigned to each such apex is $c_{1}$ (to be determined later); for notational convenience, and in order to give an idea of what kind of segments we are expecting to get per type of literal, in what follows we write $x$-segment $(i, j, k)$ to denote that $\phi(i, j, k)=c_{1}$ and that we are later going to use scaffolding segments to ensure the existence of an $x$-segment only (and analogously for $y$ and $z$-segments).

- $\operatorname{pos}\left(x_{i}\right) \Rightarrow y$-segment $(i+1, \hat{n}+2, \hat{n}+2+l)$ and $z$-segment $(i+1, \hat{n}+2+m+l, \hat{n}+2)$ $n e g\left(x_{i}\right) \Rightarrow z$-segment $(i+1, \hat{n}+2+l, \hat{n}+2)$ and $y$-segment $(i+1, \hat{n}+2, \hat{n}+2+m+l)$

- $\operatorname{pos}\left(y_{j}\right) \Rightarrow z$-segment $(\hat{n}+2+l, j+1, \hat{n}+2)$ and $x$-segment $(\hat{n}+2, j+1, \hat{n}+2+m+l)$ $n e g\left(y_{j}\right) \Rightarrow x$-segment $(\hat{n}+2, j+1, \hat{n}+2+l)$ and $z$-segment $(\hat{n}+2+m+l, j+1, \hat{n}+2)$

- $\operatorname{pos}\left(z_{k}\right) \Rightarrow x$-segment $(\hat{n}+2, \hat{n}+2+l, k+1)$ and $y$-segment $(\hat{n}+2+m+l, \hat{n}+2, k+1)$ $n e g\left(z_{k}\right) \Rightarrow y$-segment $(\hat{n}+2+l, \hat{n}+2, k+1)$ and $x$-segment $(\hat{n}+2, \hat{n}+2+m+l, k+1)$

\footnotetext{
${ }^{3}$ The support is actually a subset of this; these are all the points (bidder valuations) with potentially non-zero probabilities. This will become clear in the actual construction.
} 
Note that every positive occurrence of a variable of type, say, $x$ results in the following two segments: a negative literal segment starting at $(i+1, \hat{n}+2, \hat{n}+2+l)$ that intersects with the corresponding clause segment starting at $(1, \hat{n}+2+l, \hat{n}+2+l)$ (see next paragraph), and a literal segment starting at $(i+1, \hat{n}+2+m+l, \hat{n}+2)$ that does not intersect with any clause segment; this is also called a dummy-positive literal segment. Negative occurrences of variables analogously result in positive and dummy-negative literal segments; Figure 7 provided an illustration, minus the dummy segments. Dummy literal segments are introduced because (for reasons that will become apparent in the proof Lemma 6.9) we want to ensure the following:

Fact 6.7. The instance of 3SEGMENTS constructed has an equal number of positive and negative literal segments when dummies are included.

The point masses described above are depicted at Figure 9 for variables of type $z$. (The reader should ignore for now the $c_{4}$ entries of Figure 9 which correspond to scaffolding segments.)

Clause segments. The point masses below (with the appropriate usage of scaffolding segments to be specified later) will give rise to an $x$-segment, a $y$-segment and a $z$-segment, which are the clause segments of the variable of type $x, y$ and $z$ respectively, for clause $l$.

$$
\phi(1, \hat{n}+2+l, \hat{n}+2+l)=\phi(\hat{n}+2+l, 1, \hat{n}+2+l)=\phi(\hat{n}+2+l, \hat{n}+2+l, 1)=c_{2}
$$

The point masses for clause segments of variables of type $z$ are depicted at Figure 10. (The reader should ignore for now the $c_{3}$ entries of Figure 10 which correspond to scaffolding segments.)

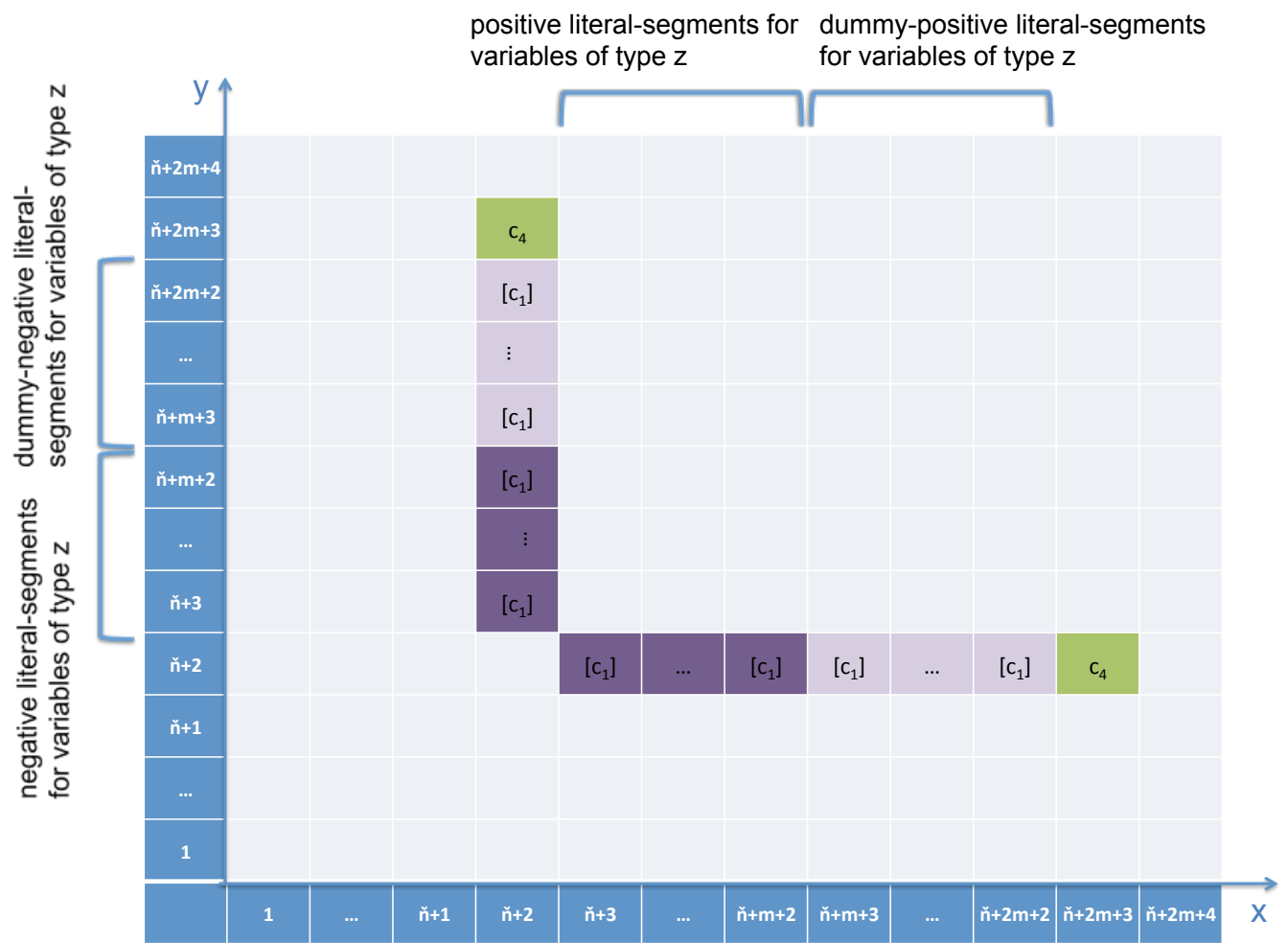

Figure 9: The plane $z=k+1, k=1, \ldots, n_{z}$, contains the literal segments of variable $z_{k}$. By [.] we mean that this point does not appear at all levels $z$. Every such level must have an equal number of $c_{1}$-entries in line $\hat{n}+2$ and column $\hat{n}+2$; also, since every variable appears at most 7 times, there can be at most 7 entries in line and column $\hat{n}+2$ for each such level. 


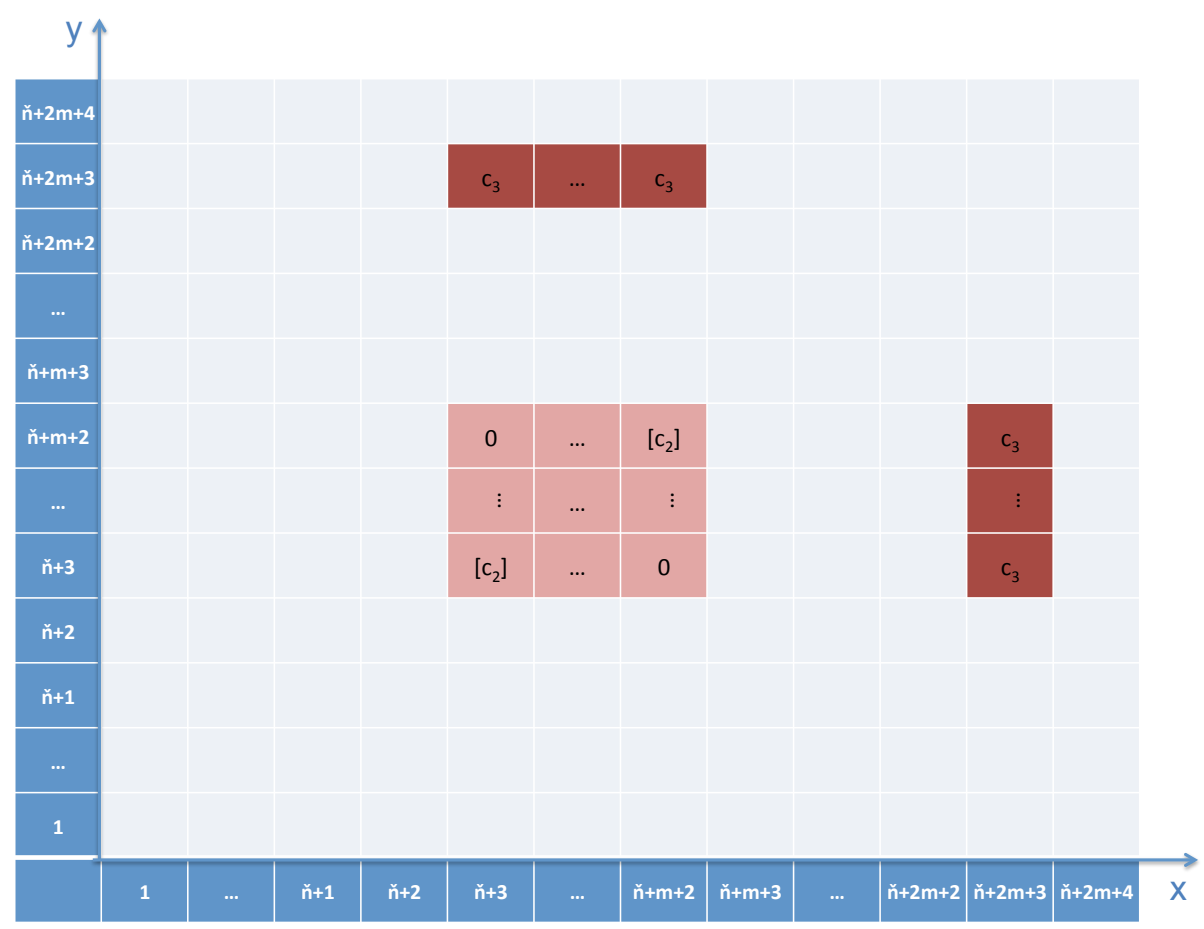

Figure 10: The plane $z=1$. Every time a variable $z_{k}$ appears in clause $l$, we introduce two literal segments lying on level $z=k+1$, through $c_{1}$ points, and a clause segment perpendicular to this plane, through a $c_{2}$ point at $(l, l, 1)$, so that it intersects with the non-dummy literal segment. The only reason why a $c_{2}$ point might be missing is because of a clause of the form $\left(x_{i} \vee y_{j}\right)$.

An example. To better understand the translation of a Typed3SAT formula into a 3SEgMENTS instance, we consider the following example:

$$
\left(x_{1} \vee y_{1} \vee \bar{z}_{1}\right) \wedge\left(\bar{x}_{1} \vee \bar{y}_{1}\right) \wedge\left(x_{2} \vee y_{1} \vee z_{1}\right)
$$

According to the construction above, the probability masses of the regular and dummy literal segments ( $c_{1}$ probability mass) and of the clause segments ( $c_{2}$ probability mass) are assigned to the coordinates displayed in the following table.

\begin{tabular}{|c||c|c|c||c|c||c|c|c|}
\hline \multicolumn{1}{|c||}{} & \multicolumn{3}{c||}{$x_{1} \vee y_{1} \vee \bar{z}_{1}$} & \multicolumn{2}{c||}{$\bar{x}_{1} \vee \bar{y}_{1}$} & \multicolumn{3}{c|}{$x_{2} \vee y_{1} \vee z_{1}$} \\
\hline & $x_{1}$ & $y_{1}$ & $\bar{z}_{1}$ & $\bar{x}_{1}$ & $\bar{y}_{1}$ & $x_{2}$ & $y_{1}$ & $z_{1}$ \\
\hline regular & $(2,4,5)$ & $(5,2,4)$ & $(5,4,2)$ & $(2,6,4)$ & $(4,2,6)$ & $(3,4,7)$ & $(7,2,4)$ & $(4,7,2)$ \\
\hline dummy & $(2,8,4)$ & $(4,2,8)$ & $(4,8,2)$ & $(2,4,9)$ & $(9,2,4)$ & $(3,10,4)$ & $(4,2,10)$ & $(10,4,2)$ \\
\hline clause & \multicolumn{2}{|c||}{$(1,5,5),(5,1,5),(5,5,1)$} & \multicolumn{1}{|c|}{$(1,6,6),(6,1,6),(6,6,1)$} & \multicolumn{2}{c|}{$(1,7,7),(7,1,7),(7,7,1)$} \\
\hline
\end{tabular}

The resulting set of segments per clause (after the application of appropriate scaffolding segments which will be explained in the next paragraph) is depicted in Figure 12 at Appendix A. Figure 13 displays the full set of segments (minus the scaffolding segments) for this formula.

Scaffolding segments. The reduction relies on the following fact:

Fact 6.8. The only intersections involving literal and clause segments will be between literal segments of literals that are negations of each other, between clause segments of the same clause and between clause segments and their corresponding literal segments.

To ensure this, we need the aforementioned scaffolding segments; these will ensure that the only segments starting from the $c_{1}$ and $c_{2}$ points specified in the previous paragraphs will indeed be the desired literal and clause segments mentioned above. 
$c_{3}$ : We first ensure that there is exactly one segment starting from every point $c_{2}$, which is perpendicular to the plane $x$ (or $y, z)=1$; in other words we ensure that there are no other segments starting at $c_{2}$ that lie on the plane, by introducing: $\phi(1, \hat{n}+l+2, \hat{n}+2 m+3)=$ $\phi(1, \hat{n}+2 m+3, \hat{n}+l+2)=\phi(\hat{n}+l+2,1, \hat{n}+2 m+3)=\phi(\hat{n}+2 m+3,1, \hat{n}+l+2)=$ $\phi(\hat{n}+l+2, \hat{n}+2 m+3,1)=\phi(\hat{n}+2 m+3, \hat{n}+l+2,1)=c_{3}$, for all $l=1, \ldots, m$; see Figure 10 for an illustration. In order to force the marginal profit contribution function to be zero at a $c_{2}$ point for some appropriately chosen bidder/direction, we impose the requirement that:

$$
s(\hat{n}+l+2) \cdot\left(c_{2}+c_{3}\right) \leq s(\hat{n}+2 m+3) \cdot c_{3}, \forall l=1, \ldots, m
$$

For example, suppose that we want point $(1, \hat{n}+2+l, \hat{n}+2+l)$ to be the apex of an $x$-segment only, and that no $y$ or $z$-segment should start at this point. We achieve this by including the points $\phi(1, \hat{n}+l+2, \hat{n}+2 m+3)=\phi(1, \hat{n}+2 m+3, \hat{n}+l+2)=c_{3}$; the above inequality then ensures that

$$
g(1, \hat{n}+2+l, \hat{n}+2+l)=h(1, \hat{n}+2+l, \hat{n}+2+l)=0
$$

and therefore there are no $y$ or $z$-segments starting at this point.

$c_{4}$ : We next ensure that there are no segments starting from $c_{1}$ points that go along row or column $\hat{n}+2$, by introducing: $\phi(i+1, \hat{n}+2, \hat{n}+2 m+3)=\phi(i+1, \hat{n}+2 m+3, \hat{n}+2)=$ $\phi(\hat{n}+2, j+1, \hat{n}+2 m+3)=\phi(\hat{n}+2 m+3, j+1, \hat{n}+2)=\phi(\hat{n}+2, \hat{n}+2 m+3, k+1)=$ $\phi(\hat{n}+2 m+3, \hat{n}+2, k+1)=c_{4}$, for $i=1, \ldots, n_{x}, j=1, \ldots, n_{y}, k=1, \ldots, n_{z}$; see Figure 9 for an illustration. Since we have at most 7 occurrences of any variable, and hence at most $7 c_{1}$-entries on any row or column $\hat{n}+2$ at a given level, the requirement we impose (along the same lines as above) is that:

$$
s(\hat{n}+l+2) \cdot\left(7 c_{1}+c_{4}\right) \leq s(\hat{n}+2 m+3) \cdot c_{4}, \forall l=1, \ldots, 2 m
$$

$c_{5}$ : Finally, we ensure that there are no $x$-segments (resp. $y$-segments, $z$-segments) starting from a point $c_{1}$ on plane $x=i+1$ (resp. $y=i+1, z=i+1$ ) for $i=1, \ldots, \hat{n}$, by introducing: $\phi(\hat{n}+2 m+4, l, \hat{n}+2)=\phi(\hat{n}+2 m+4, \hat{n}+2, l)=c_{5}(\operatorname{resp} . \phi(\hat{n}+2, \hat{n}+2 m+4, l)=$ $\left.\phi(l, \hat{n}+2 m+4, \hat{n}+2)=c_{5}, \phi(l, \hat{n}+2, \hat{n}+2 m+4)=\phi(\hat{n}+2, l, \hat{n}+2 m+4)=c_{5}\right)$ for all $l=\hat{n}+3, \ldots, \hat{n}+2 m+2$; see Figure 11 for an illustration. Noticing that $c_{1}$ can appear at position $(i+1, l, \hat{n}+2)$ only for one $i \in\{1,2, \ldots, \hat{n}\}$ (and analogously for the other positions of $c_{5}$ points) it suffices to impose the requirement that:

$$
s(i+1) \cdot\left(c_{1}+c_{5}\right) \leq s(\hat{n}+2 m+4) \cdot c_{5}, \forall i=1, \ldots, \hat{n}
$$

There is one final issue to address: from each of the scaffolding points defined above, there is not one, but three segments starting from it. Since the scaffolding segments do not intersect with any of the literal and clause segments but only with each other, for the purposes of our reduction it suffices to consider the most profitable set of scaffolding segments we can include in our solution: this way, when we prove our reduction's guarantees, we can make sure to tune the target profit in a way that will ensure the inclusion in the optimal solution of this most profitable set, so that we won't have to worry about the other scaffolding segments. In the remaining of this paragraph we will point out the most profitable set of segments in each of the three cases above; to facilitate the exposition we will move across planes $z=1, \ldots, \hat{n}+2 m+4$, so that the reader can consult Figures 9, 10 and 11 along the way.

Starting with plane $z=1$ and the $c_{3}$ scaffolding points, we will focus wlog on the points $(\hat{n}+2+l, \hat{n}+2 m+3,1), l=1, \ldots, m$, the apices of an $x$-segment of weight $s(\hat{n}+2+l)$. $(m-l+1) c_{3}$, a $y$-segment of weight $s(\hat{n}+2 m+3) \cdot c_{3}$ and a $z$-segment of weight $s(1) \cdot c_{3}$. The $y$-segment of every point clearly dominates the $z$-segment of the same point, while the whole set of all $m y$-segments from all such points (with total weight $\left.m \cdot s(\hat{n}+2 m+3) \cdot c_{3}\right)$ is better than any combination of $l y$-segments followed by a (long) $x$-segment (with total weight $\left.l \cdot s(\hat{n}+2 m+3) \cdot c_{3}+s(\hat{n}+2+l) \cdot(m-l) c_{3}\right)$. Therefore, the most profitable set of segments consists of $m y$-segments, each with a weight of $s(\hat{n}+2 m+3) \cdot c_{3}$. 
Moving on to planes $z=k+1, k=1 \ldots n_{z}$ and the $c_{4}$ scaffolding points, we will focus wlog on the points $(\hat{n}+2, \hat{n}+2 m+3, k+1)$, the apices of an $x$-segment of weight $s(\hat{n}+2) \cdot c_{4}$, a $y$-segment of weight $s(\hat{n}+2 m+3) \cdot c_{4}$ and a $z$-segment with weight $s(k+1) \cdot\left(n_{z}-k+1\right) c_{4}$. The $y$-segment of every point clearly dominates the $x$-segment of the same point, while the whole set of all $n_{z} y$-segments from all such points (with total weight $n_{z} \cdot s(\hat{n}+2 m+3) \cdot c_{4}$ ) is better than any combination of $k y$-segments followed by a (long) $z$-segment (with total weight $\left.k \cdot s(\hat{n}+2 m+3) \cdot c_{4}+s(k+1) \cdot\left(n_{z}-k\right) c_{4}\right)$. Therefore, the most profitable set of segments consists of $n_{z} y$-segments, each with a weight of $s(\hat{n}+2 m+3) \cdot c_{4}$.

Finally, for plane $z=\hat{n}+2 m+4$ and the $c_{5}$ scaffolding points, we will focus wlog on the points $(\hat{n}+2, \hat{n}+2+l, \hat{n}+2 m+4), l=1, \ldots, 2 m$, the apices of an $x$-segment of weight $s(\hat{n}+2) \cdot c_{5}$, a $y$-segment of weight $s(\hat{n}+2+l) \cdot(2 m-l+1) c_{5}$ and a $z$-segment with weight $s(\hat{n}+2 m+4) \cdot c_{5}$. The $z$-segment of every point clearly dominates the $x$-segment of the same point, while the whole set of all $2 m z$-segments from all such points (with total weight $2 m \cdot s(\hat{n}+2 m+4) \cdot c_{5}$ ) is better than any combination of $l z$-segments followed by a (long) $y$-segment (with total weight $\left.l \cdot s(\hat{n}+2 m+4) \cdot c_{5}+s(\hat{n}+2+l) \cdot(2 m-l) c_{5}\right)$. Therefore, the most profitable set of segments consists of $2 m z$-segments, each with a weight of $s(\hat{n}+2 m+4) \cdot c_{5}$.

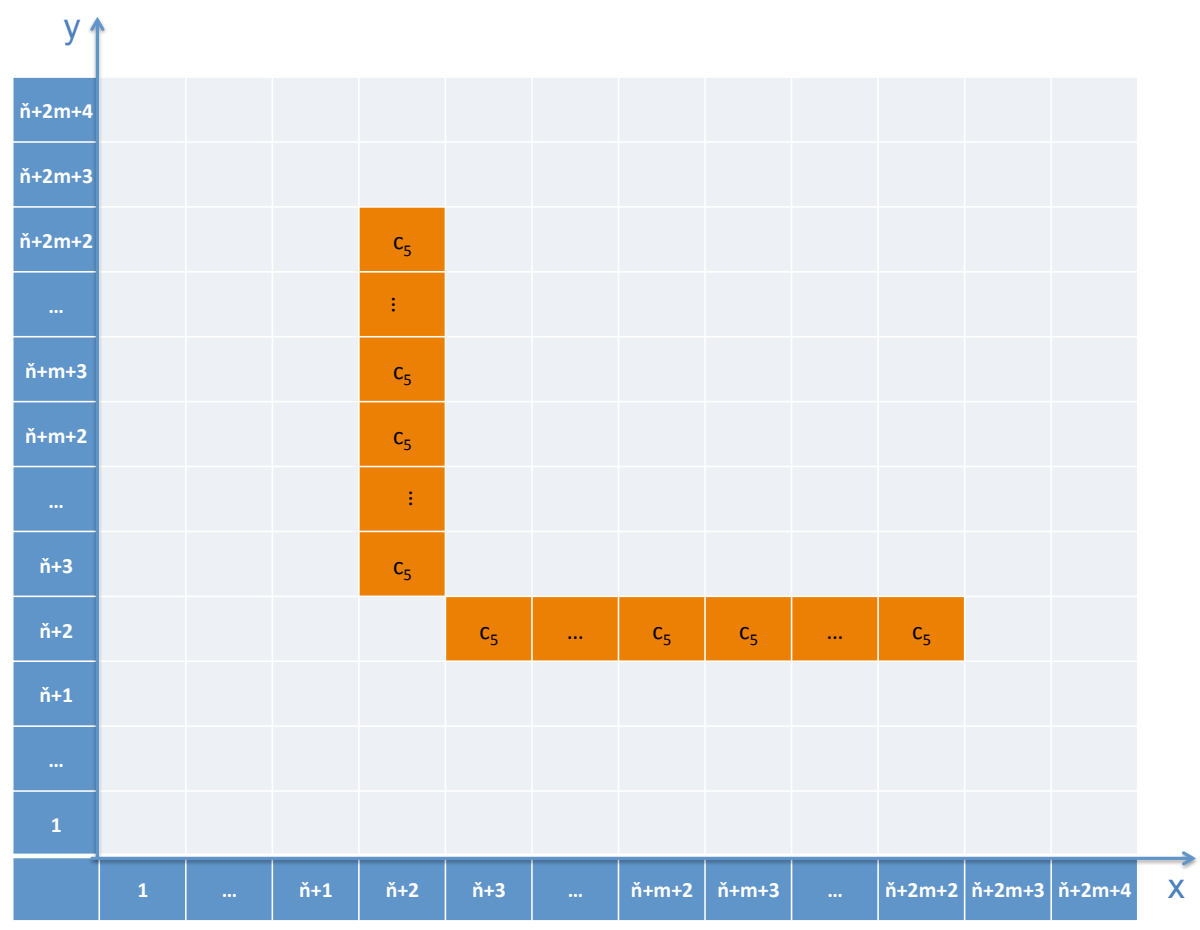

Figure 11: The plane $z=\hat{n}+2 m+4$.

The support. We are now ready to set the values of the support $s(\cdot)$ and of the variables $c_{1}, \ldots, c_{5}$. Those values will need to satisfy the aforementioned constraints $(5,6,7)$, and since $s(\cdot)$ is increasing, it actually suffices to satisfy the following stronger constraints:

$$
\begin{aligned}
& (5) \Leftarrow s(\hat{n}+2 m+2) \cdot\left(c_{2}+c_{3}\right) \leq s(\hat{n}+2 m+3) \cdot c_{3} \\
& (6) \Leftarrow s(\hat{n}+2 m+2) \cdot\left(7 c_{1}+c_{4}\right) \leq s(\hat{n}+2 m+3) \cdot c_{4} \\
& (7) \Leftarrow s(\hat{n}+2 m+2) \cdot\left(c_{1}+c_{5}\right) \leq s(\hat{n}+2 m+4) \cdot c_{5}
\end{aligned}
$$

Moreover, the values chosen need to satisfy the following inequality, which we will use later in the proof of soundness of our reduction:

$$
m \cdot s(1) c_{2} \leq \bar{n} \cdot s(\hat{n}+2) c_{1}
$$


where $\bar{n}$ denotes the total number of literal occurrences in the formula.

It is easy to check that if we pick the values for $s(\cdot)$ as follows:

$$
s(i)=\left\{\begin{array}{ll}
1+\frac{i-1}{\hat{n}+2 m+1} & \text { for } i=1, \ldots, \hat{n}+2 m+2 \\
4 & \text { for } i=\hat{n}+2 m+3 \\
5 & \text { for } i=\hat{n}+2 m+4
\end{array},\right.
$$

then constraints $(8,9,10,11)$ above are all satisfied with equality, as long as the variables $c_{1}, \ldots, c_{5}$ satisfy the following system (where we can choose the value $c_{1}$ so that all of the entries across $\phi(\cdot, \cdot, \cdot)$ sum up to 1 and we get a proper probability distribution):

$$
\begin{aligned}
c_{2} & =\frac{\bar{n}}{m}\left(1+\frac{\hat{n}+1}{\hat{n}+2 m+1}\right) c_{1} \\
c_{3} & =c_{2} \\
c_{4} & =7 c_{1} \\
c_{5} & =\frac{2}{3} c_{1}
\end{aligned}
$$

We are now ready to provide completeness and soundness guarantees for our reduction.

Lemma 6.9. The construction described above is an approximation-preserving reduction from TyPeD3SAT to 3SEgments.

Proof. We first note that regardless of the instance of TyPED3SAT we are reducing from, we can always obtain a fixed profit for 3SEGMENTs from the scaffolding segments; by picking the most profitable set of segments, as discussed above, we get a total profit of:

$$
F=6 m \cdot s(\hat{n}+2 m+3) c_{3}+2 n \cdot s(\hat{n}+2 m+3) c_{4}+12 m \cdot s(\hat{n}+2 m+4) c_{5}
$$

Let $\bar{n}$ be the total number of literal occurrences in the formula. We then have the following:

- If the TyPed3SAT formula is satisfiable then the optimal profit of 3SEGMENTs is exactly:

$$
m \cdot s(1) c_{2}+\bar{n} \cdot s(\hat{n}+2) c_{1}+F
$$

To see this first consider the following way to pick the literal segments according to the truth values assigned to the corresponding variables: if a variable is set to true ${ }^{4}$ we include its positive literal segments (both regular and dummies). Notice that -thanks to the dummy literal segments- there is an equal number of positive and negative literal segments overall (dummies included), with a total of $2 \bar{n}$ of them (see Fact 6.7 ); we include exactly half of them for every variable (either the positive or the negative ones), so the total profit from these segments is exactly $\bar{n} \cdot s(\hat{n}+2) c_{1}$.

Moreover, since the formula is satisfiable, at least one literal per clause is satisfied; if this is a positive ${ }^{4}$ literal, then the variable has been set to true and the literal segments for this variable included in our 3SEgments solution will be the positive ones. Furthermore, since the variable appears as a positive literal at this clause, our construction ensures that the corresponding clause segment intersects only with the negative literal segment of this variable (see Fact 6.8); since we have already included the positive literal segment in our solution, the negative literal segment is not included, and we are therefore free to include the clause segment in our solution as well. Each one of these clause segments contributes $s(1) c_{2}$; noticing that we cannot include more than one clause segment from each clause (because they intersect) we get that their total contribution is exactly $m \cdot s(1) c_{2}$.

- If the optimal assignment for TyPED3SAT satisfies at most a $(1-\epsilon)$ fraction of the clauses, then the optimal profit for 3SEGMENTS is at most:

$$
(1-\epsilon) m \cdot s(1) c_{2}+\bar{n} \cdot s(\hat{n}+2) c_{1}+F
$$

\footnotetext{
${ }^{4}$ The other case is completely symmetrical.
} 
Notice that, since the quantities $F, n, \bar{n}$ and $\hat{n}$ are all linear in $m$, this claim immediately establishes that the reduction is approximation-preserving.

To prove this we show how to transform a solution of profit $\geq(1-\epsilon) m \cdot s(1) c_{2}+\bar{n} \cdot s(\hat{n}+$ $2) c_{1}+F$ to a truth assignment that satisfies more than an $(1-\epsilon)$ fraction of the clauses. First notice that, wlog, any solution of 3SEGMENTs that achieves this profit will always include the most profitable set of scaffolding segments discussed above: those segments do not intersect with any literal or clause segments, so it makes no sense not to include them. Clause and literal segments do intersect with each other, however in order to achieve the additional profit of $\bar{n} \cdot s(\hat{n}+2) c_{1}$, we need to include exactly $\bar{n}$ literal segments: Obviously we cannot include any more literal segments without having intersections between them. More importantly though, we cannot substitute any literal segments for clause segments, since, even if we included one clause segment per clause (remember that all clause segments of a given clause intersect with each other), and skipped some of the literal segments, we would only get a profit of $m \cdot s(1) c_{2}$ which, as (11) guarantees, is less than the required profit $\bar{n} \cdot s(\hat{n}+2) c_{1}$. Finally, we include exactly one clause segment per clause for a certain fraction of the clauses: we cannot include more than one clause segment per clause without having intersecting clause segments.

The $\bar{n}$ literal segments that are included in our solution correspond to a truth assignment to the variables of the formula as described above: we set every variable whose positive (resp. negative) literal segments are included to true (resp. false). The claim follows by noticing that this truth assignment satisfies every clause for which a clause segment was included in the 3SEGMENTS solution: indeed, if a clause segment is included (for example corresponding to a literal of the form $\bar{x}_{3}$ ), then its (intersecting) positive literal segment $x_{3}$ cannot be included, and therefore the negative literal segment of $\bar{x}_{3}$ (which in turn intersects with the positive literal segment $x_{3}$ ) can be included, resulting to an assignment of false to $x_{3}$, which satisfies the clause under consideration.

To conclude the proof, we note that if the fraction of the clauses for which we included a clause segment is more than $(1-\epsilon)$ of the clauses, this means that this particular truth assignment satisfies more than a $(1-\epsilon)$ fraction of the clauses of the original formula, a contradiction.

Combining Lemmas 6.4 and 6.9 immediately yields our main theorem for this section:

Theorem 6.10. 3OptimalAuctionDesign is APX-complete.

\section{Discussion and Open Problems}

Even though in this paper we focused on deterministic mechanisms, our geometric characterization has interesting consequences for randomized mechanisms as well. Remember that for the discrete case the optimal (deterministic) mechanism immediately follows from solving the integer program of Problem $C$ in Section 5.1, i.e. computing a maximum weight independent set in the corresponding graph. Our first observation is that the linear programming relaxation of this integer program corresponds to computing the optimal randomized mechanism. For two bidders, where the graph is bipartite and the integer program is totally unimodular, the optimal integer solution is also the optimal of the relaxed linear program. Therefore, for two bidders, the program of Problem $C$ computes a deterministic mechanism that is optimal among all randomized mechanisms: this is reminiscent of Myerson's original result, where the deterministic mechanism obtained is optimal for the (larger) class of Bayesian truthful randomized mechanisms [21]. For a constant number of three or more bidders, the appropriate generalization of our geometric characterization (which was not discussed at length in this paper) that yields polynomial-time algorithms for computing the optimal randomized auction, in sharp contrast with the intractability of computing the optimal deterministic auction, already from three bidders; of course, for a large number of bidders the size of this linear program may become exponentially large and therefore this approach is infeasible. For an alternative linear program 
that computes the optimal randomized auction for any number of bidders, when the distribution is given explicitly, the reader is referred to [11].

The main insight in deriving the auction for two bidders is the connection of the discrete case with the weighted independent set problem in a bipartite graph. To derive the solution to the continuous case, we resorted to a long-winded proof of a duality theorem. We believe the theorem is quite interesting in its own right, but the question remains: is there a direct argument, through a simple quantization of the distribution? There is a technical problem which has thwarted our attempts at such a direct proof, relating to the fact that the curves in Figure 1 may not be Lipschitz continuous. We do not know whether this obstacle is real (that is, whether there are Lipschitz continuous distributions that yield optimal auctions that are not Lipschitz continuous), or whether such a more direct proof is ultimately possible.

Our APX-completeness proof establishes that there is some constant lower bound on the approximability of the optimal auction design problem. An important open problem of this work is to close the gap between the best approximation algorithm known for the optimal auction problem (currently .60) [11] and this tiny constant. We believe that progress there is attainable, by more sophisticated reductions. Our work does imply an approximation of $2 / n$ for $n$ bidders: before having the bidders announce their bids, the auctioneer looks at their joint distribution and privately runs the optimal auction for all possible pairs of bidders. Since solving for the optimal auction is nothing but a maximum weight independent set problem on the corresponding graph, it is easy to prove that the profit of the best of those $\left(\begin{array}{l}n \\ 2\end{array}\right)$ auctions is at least $2 / n$ of the overall profit. The auctioneer then rejects a priori all but the bidders who were part of the most profitable two-bidder auction and then runs it. The overall auction is obviously truthful as long as bidders are rejected before even submitting their bids. For 3 bidders this gives an approximation ratio of 2/3, improving over Ronen's auction [27], but for $n=4$ already, the approximation ratio drops below $1 / 2$.

Acknowledgements. We would like to thank the reviewers for constructive feedback and for pointing out an error in an earlier version of the paper, as well as the STOC reviewers for their useful comments on the conference version of the paper. CP wants to thank Benny Moldovanu for encouraging him and inspiring him to look at this problem. We also thank Adam Szeidl, Jason Hartline and Tim Roughgarden for interesting feedback and Shahar Dobzinski, $\mathrm{Hu} \mathrm{Fu}$ and Bobby Kleinberg for their comments and for sharing with us an early manuscript of their work [11].

\section{References}

[1] Mark Armstrong. Optimal multi-object auctions. Review of Economic Studies, 67(3):45581, July 2000.

[2] Sayan Bhattacharya, Gagan Goel, Sreenivas Gollapudi, and Kamesh Munagala. Budget constrained auctions with heterogeneous items. In STOC, pages 379-388, 2010.

[3] Jeremy Bulow and John Roberts. The simple economics of optimal auctions. Journal of Political Economy, 97(5):1060-90, October 1989.

[4] Shuchi Chawla, Jason D. Hartline, and Robert D. Kleinberg. Algorithmic pricing via virtual valuations. In ACM Conference on Electronic Commerce, pages 243-251, 2007.

[5] Shuchi Chawla, Jason D. Hartline, David L. Malec, and Balasubramanian Sivan. Multiparameter mechanism design and sequential posted pricing. In STOC, pages 311-320, 2010.

[6] Xi Chen and Xiaotie Deng. Settling the complexity of two-player nash equilibrium. In FOCS, pages 261-272, 2006.

[7] Jacques Cremer and Richard P McLean. Optimal selling strategies under uncertainty for a discriminating monopolist when demands are interdependent. Econometrica, 53(2):345-61, March 1985.

[8] Jacques Cremer and Richard P McLean. Full extraction of the surplus in bayesian and dominant strategy auctions. Econometrica, 56(6):1247-57, November 1988. 
[9] Constantinos Daskalakis, Paul W. Goldberg, and Christos H. Papadimitriou. The complexity of computing a nash equilibrium. In STOC, pages 71-78, 2006.

[10] Peerapong Dhangwatnotai, Tim Roughgarden, and Qiqi Yan. Revenue maximization with a single sample. In ACM Conference on Electronic Commerce, pages 129-138, 2010.

[11] Shahar Dobzinski, Hu Fu, and Robert Kleinberg. Optimal auctions with correlated bidders are easy. In $S T O C, 2011$.

[12] Lawrence C. Evans. Partial differential equations and monge-kantorovich mass transfer (surveypaper). In Current Developments in Mathematics, 1997, International Press, 1999.

[13] Andrew V. Goldberg, Jason D. Hartline, Anna R. Karlin, Andrew Wright, and Michael Saks. Competitive auctions. In Games and Economic Behavior, pages 72-81, 2002.

[14] Venkatesan Guruswami, Jason D. Hartline, Anna R. Karlin, David Kempe, Claire Kenyon, and Frank McSherry. On profit-maximizing envy-free pricing. In SODA, pages 1164-1173, 2005.

[15] Jason Hartline. Lectures on approximation in mechanism design. Lecture notes, Northwestern University, 2010.

[16] Jason D. Hartline and Tim Roughgarden. Optimal mechanism design and money burning. In $S T O C$, pages 75-84, 2008.

[17] Jason D. Hartline and Tim Roughgarden. Simple versus optimal mechanisms. In ACM Conference on Electronic Commerce, pages 225-234, 2009.

[18] Philippe Jehiel, Moritz Meyer-ter Vehn, and Benny Moldovanu. Mixed bundling auctions. Journal of Economic Theory, 134(1):494-512, May 2007.

[19] Elias Koutsoupias and Christos H. Papadimitriou. Worst-case equilibria. In STACS, pages 404-413, 1999.

[20] R Preston McAfee and Philip J Reny. Correlated information and mechanism design. Econometrica, 60(2):395-421, March 1992.

[21] Roger B. Myerson. Optimal auction design. Mathematics of Operations Research, 6:58-73, 1981.

[22] Noam Nisan and Amir Ronen. Algorithmic mechanism design (extended abstract). In STOC, pages 129-140, 1999.

[23] Noam Nisan, Tim Roughgarden, Éva Tardos, and Vijay V. Vazirani. Algorithmic Game Theory. Cambridge University Press, New York, NY, USA, 2007.

[24] Christos H. Papadimitriou. Algorithms, games, and the internet. In ICALP, pages 1-3, 2001.

[25] Christos H. Papadimitriou, Michael Schapira, and Yaron Singer. On the hardness of being truthful. In FOCS, pages 250-259, 2008.

[26] Christos H. Papadimitriou and Mihalis Yannakakis. Optimization, approximation, and complexity classes. Journal of Computer and System Sciences, 43(3):425 - 440, 1991.

[27] Amir Ronen. On approximating optimal auctions. In ACM Conference on Electronic Commerce, pages 11-17, 2001.

[28] Amir Ronen and Amin Saberi. On the hardness of optimal auctions. In FOCS, pages 396-405, 2002.

[29] Tim Roughgarden. The price of anarchy is independent of the network topology. J. Comput. Syst. Sci., 67(2):341-364, 2003.

[30] Tim Roughgarden and Éva Tardos. How bad is selfish routing? J. ACM, 49(2):236-259, 2002.

[31] William Vickrey. Counterspeculation, auctions, and competitive sealed tenders. The Journal of Finance, 16(1):8-37, 1961. 


\section{A Figures for Example Typed3SAT Formula}

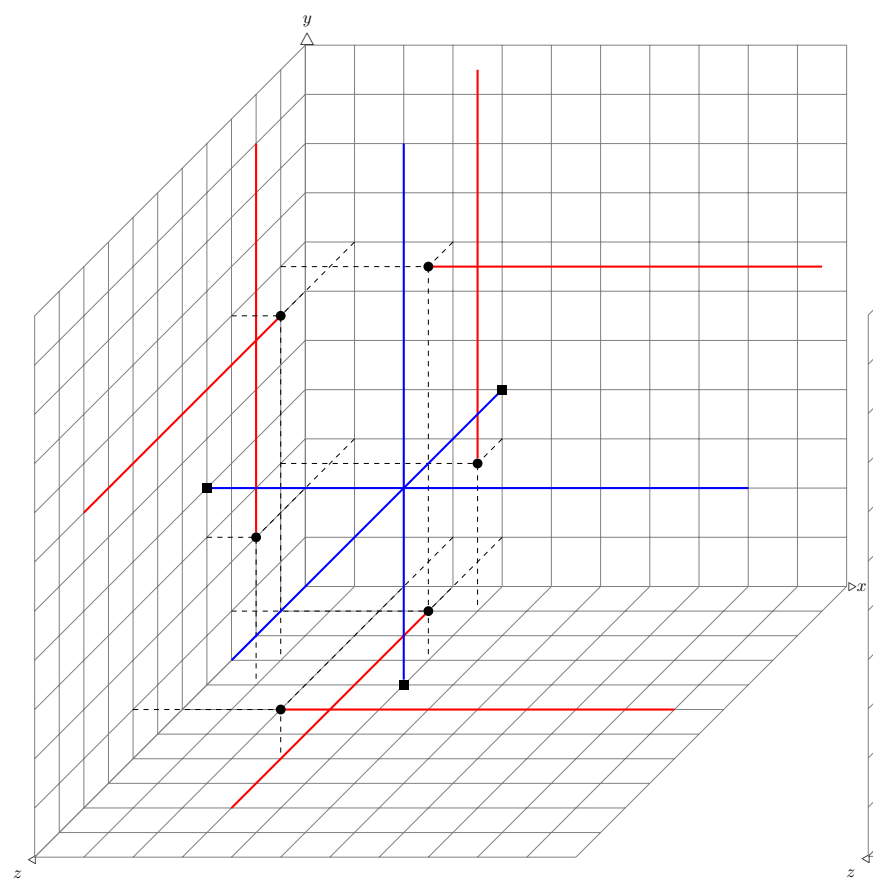

(a) The gadget for clause $x_{1} \vee y_{1} \vee \bar{z}_{1}$.

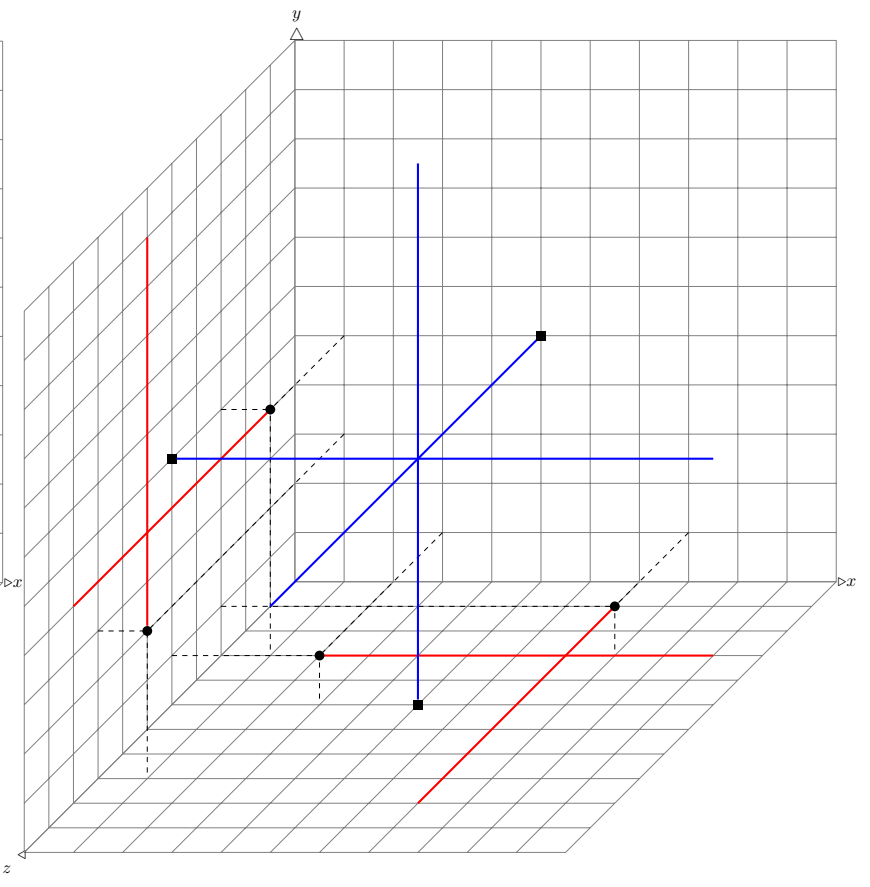

(b) The gadget for clause $\bar{x}_{1} \vee \bar{y}_{1}$.

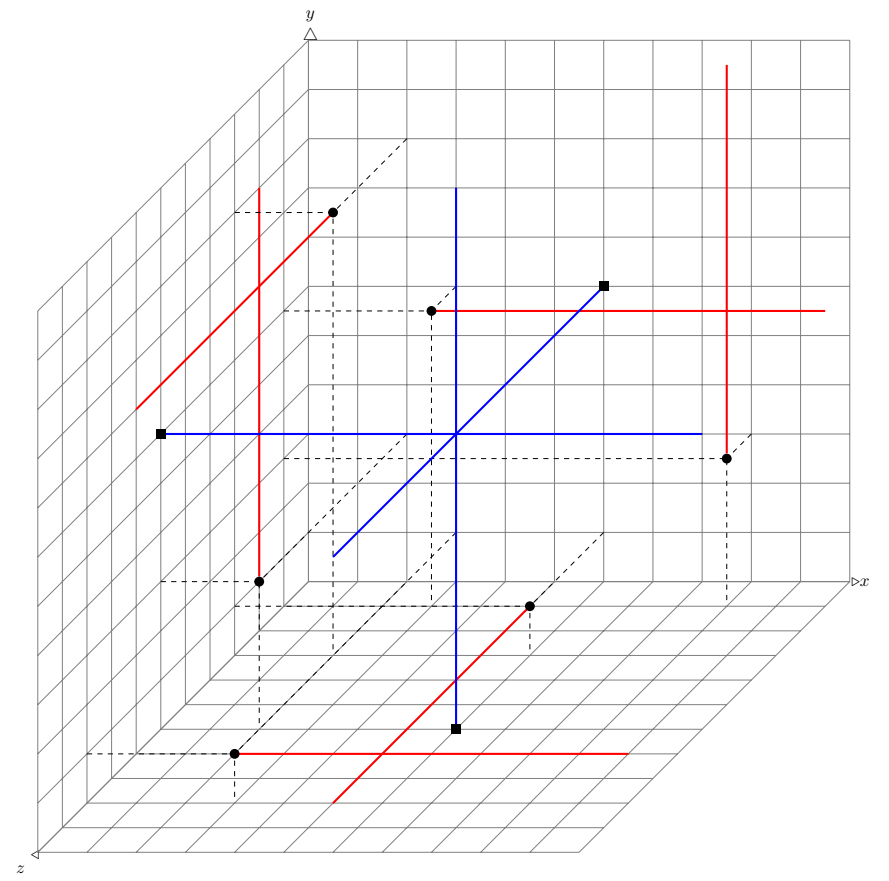

(c) The gadget for clause $x_{2} \vee y_{1} \vee z_{1}$.

Figure 12: The gadgets resulting from $\left(x_{1} \vee y_{1} \vee \bar{z}_{1}\right) \wedge\left(\bar{x}_{1} \vee \bar{y}_{1}\right) \wedge\left(x_{2} \vee y_{1} \vee z_{1}\right)$. 


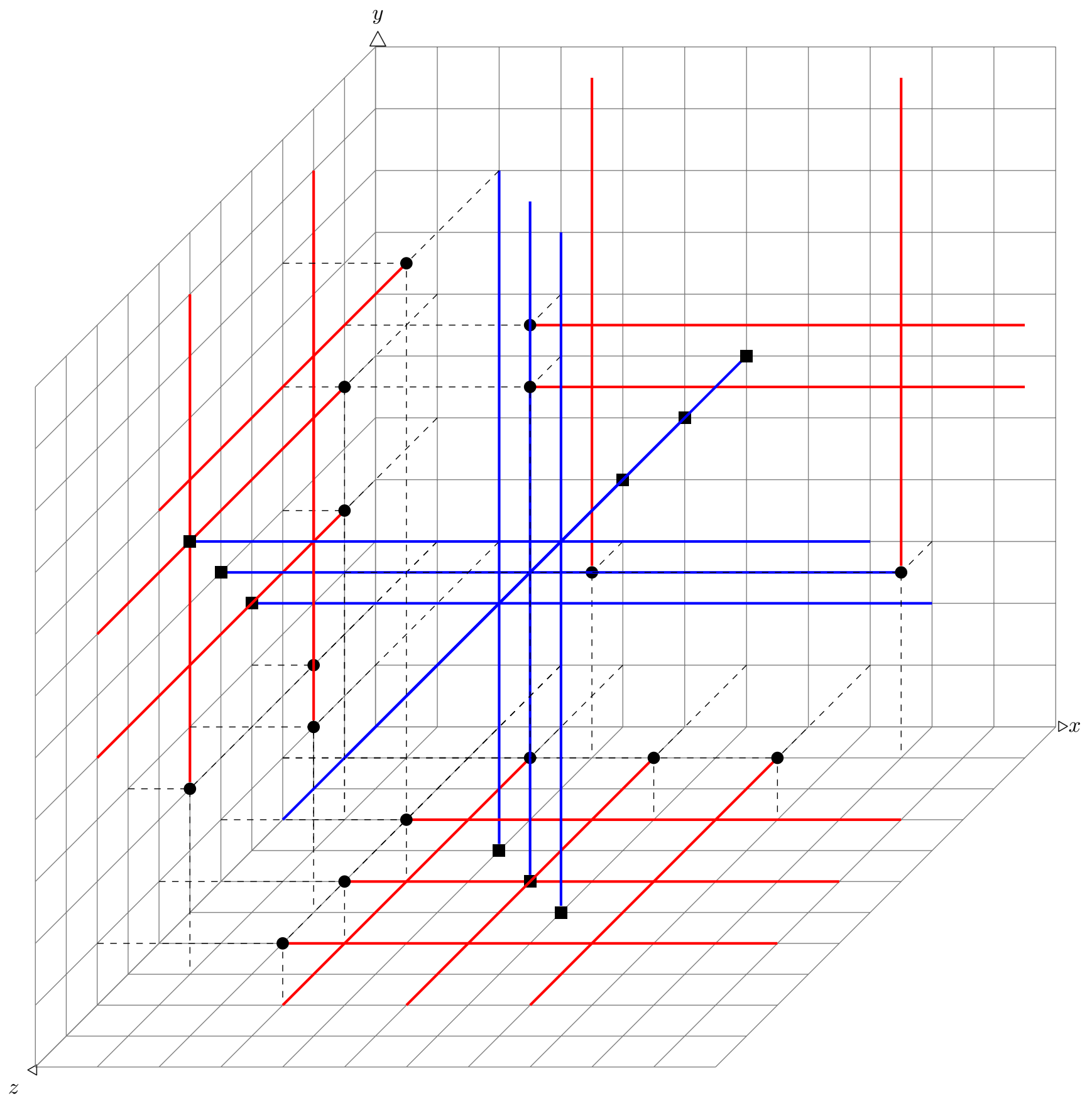

Figure 13: The full set of segments for $\left(x_{1} \vee y_{1} \vee \bar{z}_{1}\right) \wedge\left(\bar{x}_{1} \vee \bar{y}_{1}\right) \wedge\left(x_{2} \vee y_{1} \vee z_{1}\right)$. 